\title{
Phenomenology of combined resummation for Higgs and Drell-Yan
}

\section{Tanjona R. Rabemananjara}

Tif Lab, Dipartimento di Fisica, Università di Milano, and INFN, Sezione di Milano, Via Celoria 16, I-20133 Milano, Italy

E-mail: tanjona.rabemananjara@mi.infn.it

ABSTRACT: We study the phenomenological impact of a recently suggested formalism for the combination of threshold and a so-called threshold-improved transverse momentum resummation, by using it to improve the fixed-order results. This formalism allows for a systematic improvement of the transverse momentum resummation that is valid in the entire range of $p_{T}$ by the inclusion of the threshold contribution. We use the Borel method as a suitable prescription for defining the inverse Mellin and Fourier transforms in the context of combined resummed expression. The study is applied to two QCD processes, namely the Higgs boson produced via gluon fusion and $Z$ boson production via the Drell-Yan mechanism. We compare our results to the standard transverse momentum resummation, as well as to the fixed-order results. We find that the threshold-improved transverse momentum resummation leads to faster perturbative convergence at small $p_{T}$ while the inclusion of threshold resummation improves the agreement with fixed-order calculations at medium and large $p_{T}$. These effects are more pronounced in the case of Higgs which is known to have slower perturbative convergence.

KeYwords: QCD Phenomenology

ARXIV EPRINT: 2007.09164 


\section{Contents}

1 Introduction 1

2 Analytic formulation of combined resummation 2

2.1 Combined transverse momentum and threshold resummation 3

2.2 Inverse Fourier-Mellin 5

2.2.1 Contour and Borel integration $\quad 8$

2.2.2 Inverse Mellin 8

$\begin{array}{lll}2.3 & \text { PDF evolution for threshold-improved } p_{T} \text { resummation } & 9\end{array}$

$\begin{array}{llr}2.4 & \text { Finite order truncation of the resummed expression } & 12\end{array}$

3 Phenomenological studies $\quad 14$

3.1 Threshold-improved $p_{T}$ resummation in the large- $\hat{b}$ limit $\quad 14$

$\begin{array}{lll}3.2 & \text { Higgs boson production at LHC and DY production at Tevatron } & 15\end{array}$

$\begin{array}{llr}4 \text { Conclusions } & 18\end{array}$

\section{Introduction}

The current state-of-the-art accuracy in resummed calculations of transverse momentum spectra is $\mathrm{N}^{3} \mathrm{LL}$ matched with fixed-order predictions (NLO/NNLO), and has been achieved recently for a variety of processes including Higgs boson production [1-3], and Drell-Yan (DY) [4-9]. Since large logarithmic corrections present in transverse momentum and threshold resummation both originate from the emission of soft gluons, there have been attempts to construct a joint formalism that simultaneously resum logarithmic contributions that are enhanced at small $p_{T}$ and at partonic threshold [10,11]. Such a joint resummation has been successful in producing phenomenological results at NLL accuracy for various processes including Higgs [12] and vector boson production via DY mechanism [13] which has been recently extended to NNLL [14]. While the aforementioned resummations were done in Fourier-Mellin space, joint resummation in direct space has been achieved up to NNLL accuracy using SCET [15]. In these references, however, the inclusion of soft contributions were not fully complete as will be discussed in this paper.

Recently, a combination of threshold and transverse momentum resummation has been proposed in ref. [16] that has the following features: it reproduces the correct behaviour to any desired logarithmic order in the limit $p_{T} \rightarrow 0$ for fixed $x$; it reduces to threshold resummation in the soft limit $x \rightarrow 1$ for fixed $p_{T}$ up to power correction in $(1-x)$; and it reproduces the total cross section at any given logarithmic order in the threshold limit upon integration over the transverse momentum. This combined resummation relies mainly on two ingredients: (i) a modified transverse momentum resummation - that henceforth we 
call threshold-improved transverse momentum resummation - which leads to threshold resummed expression upon integration over $p_{T}$, (ii) the combination of the thresholdimproved $p_{T}$ expression with the pure threshold expression that takes into account all the logarithmic enhanced terms in the soft limit for finite $p_{T}$. Such a combined resummation is expected to allow for a systematic improvements of the transverse momentum that is valid for the entire range of $p_{T}$ but the phenomenological implications have never been studied.

Therefore, the main goal of this paper is to separately assess the effects of the modified transverse momentum and combined resummation on transverse momentum distributions. In order to do this, we have to deal with issues that arise from the particular construction of the threshold-improved transverse momentum resummation. The first concerns the issue of performing the inverse Fourier-Mellin transform. In most resummation formulae [17-30], transverse momentum resummation is performed in Fourier-Mellin $(N-b)$ space where closed expression can be found. However, in addition to the problem of Landau pole that prevents the existence of an inverse Mellin in standard $p_{T}$ resummation, thresholdimproved $p_{T}$ resummation presents additional singularities due to the interplay between the Mellin moment $N$ and the impact parameter $b$ in the argument of the logarithms. We show that this issue can be addressed by slightly modifying the Borel prescription that was recently studied in refs. [31, 32] in the context of threshold resummation for DY cross sections. The second has to do with logarithmic counting in which perturbative evolutions have to be treated in a different way to properly account for the threshold behaviours. Finally, in order to obtain valid predictions, we need to match the resummed expressions to the fixed-order results. This requires the computation of the inverse Fourier-Mellin transform order by order in the running of the coupling constant $\alpha_{s}$.

This paper is organized as follows. We begin in section 2 with a review of the combined threshold and threshold-improved $p_{T}$ resummation. We then describe the analytical procedures to address the issues introduced previously. Our phenomenological results are presented in section 3 in which we separately study the impact of the modified $p_{T}$ and combined resummation to the Higgs boson production at LHC via gluon-gluon fusion and a DY process at Tevatron. Finally, conclusions are drawn in section 4.

\section{Analytic formulation of combined resummation}

In this section, we first provide a brief review of the combined resummation introduced in ref. [16]. We then address specific issues that arise in the phenomenological studies of the modified $p_{T}$ resummation: (i) the need for a Fourier-Mellin inversion procedure, (ii) the treatment of the PDF evolution, and (iii) the matching to the fixed-order calculations.

Regardless of the fact that in this paper we are particularly interested in the phenomenological implication of the combined resummation to the case of Higgs and DY production, the expressions that follow are kept as general as possible such that they apply to general colour singlet hadronic production. 


\subsection{Combined transverse momentum and threshold resummation}

Consider the inclusive hard-scattering process $h_{1}+h_{2} \rightarrow F\left(Q, p_{T}\right)+X$, where the collision of two hadrons $h_{1}$ and $h_{2}$ produces a final-state system $F$ with an invariant mass $Q$ and a transverse momentum $p_{T}$ accompanied by an arbitrary final state $X$. As mentioned earlier, in this paper we study the case where the final state $F$ can be either a Higgs boson or a DY lepton pair via electroweak boson production. We denote by $\sqrt{s}$ and $\sqrt{\hat{s}}$ the center of mass-energy of the colliding hadrons and partons respectively.

Transverse momentum distributions factorize into a convolution between a parton luminosity and a partonic cross section which can be written in the following way

$$
\frac{1}{\tau^{\prime}} \frac{\mathrm{d} \sigma}{\mathrm{d} \xi_{p}}\left(\xi_{p}, \alpha_{s}\right)=\sum_{a, b} \int_{\tau^{\prime}}^{1} \frac{\mathrm{d} x}{x} \mathcal{L}_{a b}\left(\frac{\tau^{\prime}}{x}\right) \frac{1}{x} \frac{\mathrm{d} \hat{\sigma}_{a b}}{\mathrm{~d} \xi_{p}}\left(x, \xi_{p}, \alpha_{s}\right),
$$

where

$$
\tau^{\prime}=\frac{Q^{2}}{s}\left(\sqrt{1+\xi_{p}}+\sqrt{\xi_{p}}\right)^{2}, \quad \text { and } \quad x=\frac{Q^{2}}{\hat{s}}\left(\sqrt{1+\xi_{p}}+\sqrt{\xi_{p}}\right)^{2} .
$$

The differential $p_{T}$ distribution in eq. (2.1) has been expressed in terms of the dimensionless variable $\xi_{p}=p_{T}^{2} / Q^{2}$ and, for simplicity, the renormalization and factorization scale dependencies have been omitted. ${ }^{1}$ The sum is over the different partonic channels where $a$ and $b$ denote partons. As it turns out that in Mellin space, the convolution becomes a simple product, eq. (2.1) can be re-written as:

$$
\frac{\mathrm{d} \sigma}{\mathrm{d} \xi_{p}}\left(N, \xi_{p}, \alpha_{s}\right)=\sum_{a, b} \mathcal{L}_{a b}(N) \frac{\mathrm{d} \hat{\sigma}_{a b}}{\mathrm{~d} \xi_{p}}\left(N, \xi_{p}, \alpha_{s}\right),
$$

given that

$$
\begin{aligned}
\frac{\mathrm{d} \sigma}{\mathrm{d} \xi_{p}}\left(N, \xi_{p}, \alpha_{s}\right) & =\int_{0}^{1} \mathrm{~d} \tau^{\prime}\left(\tau^{\prime}\right)^{N-1} \frac{1}{\tau^{\prime}} \frac{\mathrm{d} \sigma}{\mathrm{d} \xi_{p}}\left(\tau, \xi_{p}, \alpha_{s}\right), \\
\frac{\mathrm{d} \hat{\sigma}_{a b}}{\mathrm{~d} \xi_{p}}\left(N, \xi_{p}, \alpha_{s}\right) & =\int_{0}^{1} \mathrm{~d} x x^{N-1} \frac{1}{x} \frac{\mathrm{d} \hat{\sigma}_{a b}}{\mathrm{~d} \xi_{p}}\left(x, \xi_{p}, \alpha_{s}\right) .
\end{aligned}
$$

Notice here that the cross section and its Mellin transform are denoted with the same symbol and only distinguished trough their arguments. It is worth stressing that the Mellin transform of the hadronic cross section in eq. (2.4a) is taken w.r.t. the scaling variable $\tau^{\prime}$ while the Mellin transform of the partonic one in eq. $(2.4 \mathrm{~b})$ is taken w.r.t. the partonic variable $x$. Thanks to this choice of variables, one can take simultaneously the Mellin and Fourier transform of the cross section. Indeed, $\tau^{\prime}$ and $p_{T}$ are independent variables as $\tau^{\prime}$ ranges from $0 \leq \tau^{\prime} \leq 1$ and $p_{T}$ ranges from $0 \leq p_{T} \leq \infty$. The available phase induced by such choice of variables is depicted in the right-hand side of figure 1.

The partonic part in eq. (2.3) is constructed by combining the threshold-improved transverse momentum, to be discussed below, with the threshold resummation. The two resummations are combined through a profile matching function which is chosen such that the combined result reproduces transverse momentum and threshold resummation at

\footnotetext{
${ }^{1}$ In ref. [16], the invariant mass is denoted by $M($ instead of $Q)$ and $Q=\left(\sqrt{M^{2}+p_{T}^{2}}+p_{T}\right)$.
} 

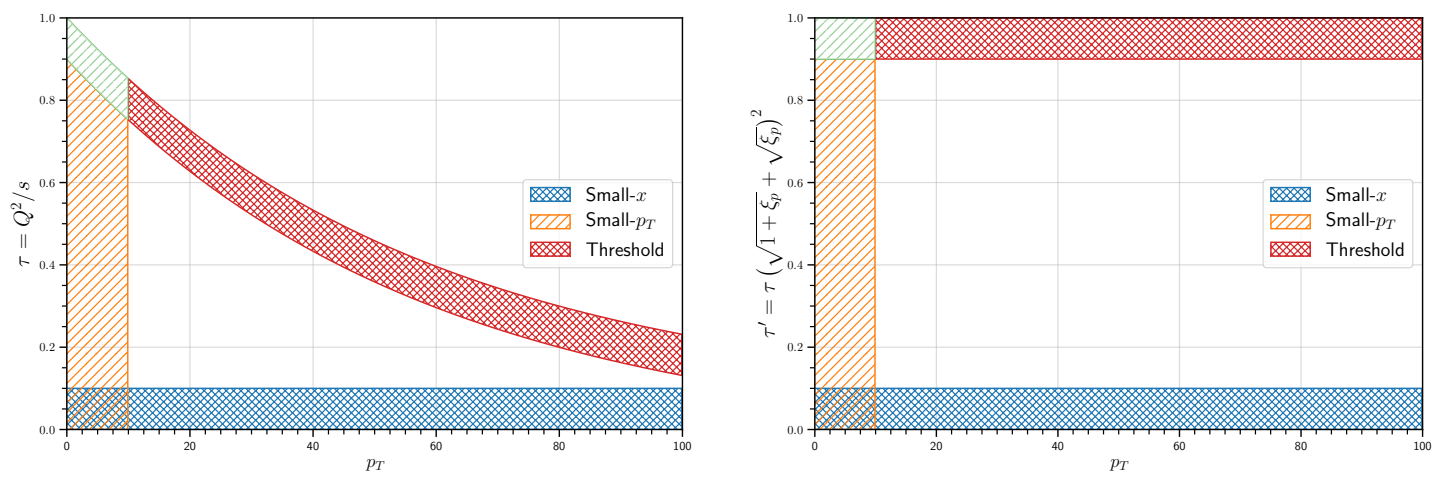

Figure 1. Available phase space for the production of a final-state system $F$ with an invariant mass $Q$ and a transverse momentum $p_{T}$ in the three kinematic limits (threshold, collinear, high-energy), parametrized in terms of the kinematic variable $\tau=Q^{2} / s$ (left) and $\tau^{\prime}$ (right).

small $p_{T}$ and large $x$ respectively. One possible expression of $\mathrm{d} \hat{\sigma}_{a b} / \mathrm{d} \xi_{p}$ has been proposed in ref. [16]

$$
\frac{\mathrm{d} \hat{\sigma}_{a b}}{\mathrm{~d} \xi_{p}}\left(N, \xi_{p}, \alpha_{s}\right)=\left(1-\mathrm{T}\left(N, \xi_{p}\right)\right) \frac{\mathrm{d} \hat{\sigma}_{a b}^{\mathrm{tr}}}{\mathrm{d} \xi_{p}}\left(N, \xi_{p}, \alpha_{s}\right)+\mathrm{T}\left(N, \xi_{p}\right) \frac{\mathrm{d} \hat{\sigma}_{a b}^{\mathrm{thrs}}}{\mathrm{d} \xi_{p}}\left(N, \xi_{p}, \alpha_{s}\right),
$$

where the profile matching function is defined as

$$
\mathrm{T}\left(N, \xi_{p}\right)=\frac{N^{k} \xi_{p}^{m}}{1+N^{k} \xi_{p}^{m}}
$$

The values of $k$ and $m$ can be chosen arbitrarily provided that $m<k$. This is because the combined resummation produces results that differ from the threshold-improved $p_{T}$ resummation by $\mathcal{O}\left(\xi_{p}^{m}\right)$ corrections when $\xi_{p} \rightarrow 0$, and from the threshold resummation by $\mathcal{O}\left(1 / N^{k}\right)$ corrections when $N \rightarrow \infty$. As mentioned in the original reference [16], combined resummation resums small- $p_{T}$ logarithms up to NNLL accuracy, while threshold ones are resummed up to NNLL* according to the counting in table 1 of ref. [33].

The threshold part $\mathrm{d} \hat{\sigma}_{a b}^{\mathrm{thrs}} / \mathrm{d} \xi_{p}$ in eq. (2.5) resums logarithms of the form $\alpha_{s}^{n} \ln ^{m}(N)$ that are enhanced in the large- $N$ limit for fixed $p_{T}$ and is given in refs. [34, 35] for Higgs and in refs. $[23,24,26,28]$ for DY production. On the other hand, $\mathrm{d} \hat{\sigma}_{a b}^{\mathrm{tr}} / \mathrm{d} \xi_{p}$ represents the improved version of the standard transverse momentum resummation from which it differs by the inclusion of soft contributions that are power-suppressed terms in the small- $p_{T}$ limit for fixed $N$. It provides a modified transverse momentum expression that reproduces threshold resummation upon integration over $p_{T}$.

While the phenomenological study of the threshold resummation does not pose new problems, technical issues arise in the modified $p_{T}$ resummation that require specific care. Hence, the following sections are mainly devoted to the threshold-improved $p_{T}$ resummation. First, in order to fully take into account the kinematics constraint on the transverse momentum conservation, it is advantageous to work in Fourier space where the part that resums small- $p_{T}$ logarithms in eq. (2.5) yields

$$
\frac{\mathrm{d} \hat{\sigma}_{a b}^{\mathrm{tr}}}{\mathrm{d} \xi_{p}}\left(N, \xi_{p}, \alpha_{s}\right)=\sigma_{0}\left(\sqrt{1+\xi_{p}}+\sqrt{\xi_{p}}\right)^{2 N} \int_{0}^{\infty} \mathrm{d} \hat{b} \frac{\hat{b}}{2} J_{0}\left(\hat{b} \sqrt{\xi_{p}}\right) \Sigma_{a b}\left(N, \lambda_{\chi}(\hat{b})\right) .
$$


In the above expression, $\sigma_{0}$ represents the leading order Born cross section which is given in [29] for Higgs and [36] for DY; $J_{0}$ is the Bessel function while $\hat{b}=b Q$ represents the modified conjugate variable to $\xi_{p}$ in Fourier space. The resummed partonic cross section is represented by $\Sigma_{a b}$ where the $\hat{b}$ dependence is embodied in $\lambda_{\chi}$ which is defined as

$$
\lambda_{\chi}(\hat{b})=\alpha_{s} \beta_{0} \ln (\chi), \quad \text { where } \quad \chi=\bar{N}^{2}+\frac{\hat{b}^{2}}{b_{0}^{2}},
$$

with the modified Mellin variable defined as $\bar{N}=N \exp \left(-\gamma_{E}\right)$ and $b_{0}=2 \exp \left(-\gamma_{E}\right)$. Notice that in comparison to the standard transverse momentum resummation, now the logarithm which is being resummed also depends on the Mellin variable $N$.

The logarithmically enhanced terms in eq. (2.7) are contained in the resummed expression $\Sigma_{a b}$ which can be organized in the following way:

$$
\Sigma_{a b}\left(N, \lambda_{\chi}\right)=\sum_{\{\mathcal{P}\}} \tilde{\mathcal{H}}_{a b}^{\{\mathcal{P}\}}(N, \chi) \exp \left(\mathcal{G}_{a b}^{\{\mathcal{P}\}}\left(N, \lambda_{\chi}\right)\right)
$$

where $\mathcal{P}$ denotes the different projectors of the LO anomalous dimension in the matrix flavour space. On one hand, the function $\mathcal{G}$ contains all the large logarithms coming from the Sudakov form factor, the evolution of the PDFs, and the evolution of the coefficient functions. Its complete expression is derived in section 2.3. On the other hand, the function $\tilde{\mathcal{H}}$ contains all the terms that behave as a constant in the large- $\hat{b}$ limit.

\subsection{Inverse Fourier-Mellin}

In order to get physical cross sections, we have to compute the inverse Fourier-Mellin transform of the $(N-b)$ space resummed expression. Mellin and/or Fourier resummed expressions generally give rise to technical complications when it comes to performing the inverse transform. Because the resummation corresponds to the asymptotic sum of a divergent series of $p_{T}$-space contributions [29], the Fourier-Mellin integral contains singularities.

In a standard procedure, Fourier and Mellin inverse transform are computed using the Minimal prescription (MP) [37]. In the case of inverse Fourier transform, this amounts to finding a $\hat{b}$ contour that avoids the singularities; while for the inverse Mellin transform, this amounts to defining an integration path that passes to the left of the branch but to the right of all singularities as shown in figure 2. This prescription defines the resummed hadronic cross section as

$$
\frac{\mathrm{d} \sigma}{\mathrm{d} \xi_{p}}\left(\xi_{p}, \alpha_{s}\right)=\frac{\tau^{\prime}}{2 \pi \mathrm{i}} \sum_{a, b} \int_{\mathrm{MP}} \mathrm{d} N\left(\tau^{\prime}\right)^{-N} \mathcal{L}_{a b}(N) \frac{\mathrm{d} \hat{\sigma}_{a b}}{\mathrm{~d} \xi_{p}}\left(N, \xi_{p}, \alpha_{s}\right) .
$$

As a result, cross sections obtained through such a procedure is finite. However, in addition to the branch cut related to the Landau pole, the integrand in eq. (2.7) contains additional singularities when

$$
\mathfrak{R e}\left(\bar{N}^{2}+\frac{\hat{b}^{2}}{b_{0}^{2}}\right) \leq 0, \quad \text { and } \quad \mathfrak{I m}\left(\bar{N}^{2}+\frac{\hat{b}^{2}}{b_{0}^{2}}\right)=0 .
$$


This means that the location of the $N$-space singularities depends on $\hat{b}$. Therefore, it is non-trivial to find a contour deformation that leaves all the singularities to the left and the branch cut to the right.

An alternative approach consists on summing the divergent series using the Borel method which was first introduced in refs. [37-39]. Recently, it was shown in ref. [31] in the context of resummation of transverse momentum distributions that the inverse FourierMellin can be performed by first resumming the divergent series using the Borel method and then removing the divergence in the inverse Borel transform. This permits the computation of the asymptotic result the divergent series is tending to. Such a procedure can be slightly modified to work with transverse momentum resummation [40]. The steps are as follows. First, we expand the resummed component $\Sigma_{a b}\left(N, \chi, \alpha_{s}\right)$ as a series in $\bar{\alpha}_{s} \ln (\chi)$ (with $\left.\bar{\alpha}_{s}=\alpha_{s} \beta_{0}\right)$ and tackle directly the divergence using the Borel summation. This then leads to a $N$-space expression that can be inverted using the MP. Hence, we have

$$
\Sigma\left(N, \lambda_{\chi}(\hat{b}), \alpha_{\mathrm{s}}\right)=\sum_{k=0}^{\infty} h_{k}\left(N, \alpha_{\mathrm{s}}\right) \bar{\alpha}_{\mathrm{s}}^{k} \ln ^{k} \chi(\hat{b})=\sum_{k=0}^{\infty} h_{k}\left(N, \alpha_{\mathrm{s}}\right) \bar{\alpha}_{\mathrm{s}}^{k} \ln ^{k}\left(\bar{N}^{2}+\frac{\hat{b}^{2}}{b_{0}^{2}}\right) .
$$

When $k=0$ the zeroth order coefficient $h_{0}\left(N, \alpha_{s}\right)$ is just a constant. Putting back the above equation into the partonic resummed expression in eq. (2.7), exchanging the integral and the sum, and finally using the trick $\mathrm{d}^{k} \chi^{\epsilon} /\left.\mathrm{d} \epsilon^{k}\right|_{\epsilon \rightarrow 0}=\ln ^{k} \chi$, we get to the following result

$$
\begin{aligned}
\frac{\mathrm{d} \hat{\sigma}^{\prime}}{\mathrm{d} \xi_{p}}\left(N, \xi_{p}, \alpha_{s}\right) & =\left.\sum_{k=0}^{\infty} h_{k}\left(N, \alpha_{s}\right) \bar{\alpha}_{\mathrm{s}}^{k} \frac{\partial^{k}}{\partial \epsilon^{k}} \int_{0}^{\infty} \mathrm{d} \hat{b} \frac{\hat{b}}{2} J_{0}\left(\hat{b} \sqrt{\xi_{p}}\right)\left(\bar{N}^{2}+\frac{\hat{b}^{2}}{b_{0}^{2}}\right)^{\epsilon}\right|_{\epsilon \rightarrow 0}, \\
& =\left.\sum_{k=0}^{\infty} h_{k}\left(N, \alpha_{s}\right) \bar{\alpha}_{\mathrm{s}}^{k} \frac{\partial^{k}}{\partial \epsilon^{k}} \frac{1}{b_{0}^{2 \epsilon}} \int_{0}^{\infty} \mathrm{d} \hat{b} \frac{\hat{b}}{2} J_{0}\left(\hat{b} \sqrt{\xi_{p}}\right)\left(4 N^{2}+\hat{b}^{2}\right)^{\epsilon}\right|_{\epsilon \rightarrow 0}
\end{aligned}
$$

Remarks are in order concerning the above equations: (i) $\mathrm{d} \hat{\sigma}^{\prime} / \mathrm{d} \xi_{p}$ is just $\mathrm{d} \hat{\sigma}^{\mathrm{tr}^{\prime}} / \mathrm{d} \xi_{p}$ without the kinematic factor $\left(\sqrt{1+\xi_{p}}+\sqrt{\xi_{p}}\right)^{2 N}$ and the Born level cross section $\sigma_{0}$, (ii) $b_{0}$ has been factorized out such that the integrand in eq. (2.13a) is a function of $N$ instead of $\bar{N}$. This allows us to perform the inverse Fourier transform analytically order by order. Hence, eq. (2.13b) becomes

$$
\frac{\mathrm{d} \hat{\sigma}^{\prime}}{\mathrm{d} \xi_{p}}\left(N, \xi_{p}, \alpha_{s}\right)=\left.\sum_{k=0}^{\infty} h_{k}\left(N, \alpha_{s}\right) \bar{\alpha}_{\mathrm{s}}^{k} \frac{\partial^{k}}{\partial \epsilon^{k}} M\left(N, \xi_{p}, \epsilon\right)\right|_{\epsilon \rightarrow 0},
$$

where the function $M$ is defined as

$$
M\left(N, \xi_{p}, \epsilon\right)=2 \mathrm{e}^{2 \gamma_{E} \epsilon}\left(\frac{N}{\sqrt{\xi_{p}}}\right)^{1+\epsilon} \frac{\mathrm{K}_{1+\epsilon}\left(2 N \sqrt{\xi_{p}}\right)}{\Gamma(-\epsilon)} .
$$

The function $\mathrm{K}$ denotes the modified Bessel function of the second kind. We can then get rid of the derivatives and the limit by performing a contour integral,

$$
\left.\frac{\partial^{k}}{\partial \epsilon^{k}} M\left(N, \xi_{p}, \epsilon\right)\right|_{\epsilon \rightarrow 0}=\frac{k !}{2 \pi \mathrm{i}} \oint_{H} \frac{\mathrm{d} \xi}{\xi^{1+k}} M\left(N, \xi_{p}, \xi\right),
$$

where $H$ represents a contour enclosing the singularity at $\xi=0$. 
Having expanded the function $\Sigma$ allowed us to perform the inverse Fourier transform, but it leaves us with a series representation of the result. Thinking of the numerical limitations of series representations, we would rather trade the sum for an integral. This basically calls for an analytic continuation, which in this case is achieved using a Borel summation and a subsequent Borel back-transformation. First, writing down the series as

$$
\frac{\mathrm{d} \hat{\sigma}^{\prime}}{\mathrm{d} \xi_{p}}\left(N, \xi_{p}, \alpha_{s}\right) \equiv A\left(\bar{\alpha}_{s}\right)=\sum_{k=0}^{\infty} h_{k}\left(N, \alpha_{s}\right) \frac{k !}{2 \pi \mathrm{i}} \oint_{H} \frac{\mathrm{d} \xi}{\xi} M\left(N, \xi_{p}, \xi\right)\left(\frac{\bar{\alpha}_{s}}{\xi}\right)^{k} .
$$

The corresponding Borel sum is then given by

$$
\begin{aligned}
\mathcal{B}[A(w)] & =\frac{1}{2 \pi \mathrm{i}} \sum_{k=0}^{\infty} h_{k}\left(N, \alpha_{\mathrm{s}}\right) \oint_{H} \frac{\mathrm{d} \xi}{\xi} M\left(N, \xi_{p}, \xi\right)\left(\frac{w}{\xi}\right)^{k} \\
& =\frac{1}{2 \pi \mathrm{i}} \oint_{H} \frac{\mathrm{d} \xi}{\xi} M\left(N, \xi_{p}, \xi\right) \Sigma\left(N, \frac{w}{\xi}, \alpha_{\mathrm{s}}\right)
\end{aligned}
$$

where we resummed back the expansion of $\Sigma$ using its definition in eq. (2.12). We finally perform the Borel back-transformation,

$$
\begin{aligned}
\frac{\mathrm{d} \hat{\sigma}^{\prime}}{\mathrm{d} \xi_{p}}\left(N, \xi_{p}, \alpha_{s}\right) & =\int_{0}^{\infty} \mathrm{d} w \mathrm{e}^{-w} \mathcal{B}\left[A\left(w \bar{\alpha}_{\mathrm{s}}\right)\right]=\frac{1}{\bar{\alpha}_{\mathrm{s}}} \int_{0}^{\infty} \mathrm{d} w^{\prime} \mathrm{e}^{-w^{\prime} / \bar{\alpha}_{\mathrm{s}}} \mathcal{B}\left[A\left(w^{\prime}\right)\right] \\
& =\frac{1}{\bar{\alpha}_{\mathrm{s}}} \int_{0}^{\infty} \mathrm{d} w \mathrm{e}^{-w / \bar{\alpha}_{\mathrm{s}}} \frac{1}{2 \pi \mathrm{i}} \oint_{H} \frac{\mathrm{d} \xi}{\xi} M\left(N, \xi_{p}, \xi\right) \Sigma\left(N, \frac{w}{\xi}, \alpha_{\mathrm{s}}\right),
\end{aligned}
$$

which represents our final resummed expression when multiplied by the factor $\sigma_{0}\left(\sqrt{1+\xi_{p}}+\right.$ $\left(\sqrt{\xi_{p}}\right)^{2 N}$ that we left out from the beginning. After cutting off the $w$-integration at some finite number $C$, we have the final expression

$$
\frac{\mathrm{d} \hat{\sigma}_{a b}^{\mathrm{tr}}}{\mathrm{d} \xi_{p}}=\frac{\sigma_{0}}{\bar{\alpha}_{\mathrm{s}}}\left(\sqrt{1+\xi_{p}}+\sqrt{\xi_{p}}\right)^{2 N} \int_{0}^{C} \mathrm{~d} w \frac{\mathrm{e}^{-w / \bar{\alpha}_{\mathrm{s}}}}{2 \pi \mathrm{i}} \oint_{H} \frac{\mathrm{d} \xi}{\xi} M\left(N, \xi_{p}, \xi\right) \Sigma_{a b}\left(N, \frac{w}{\xi}, \alpha_{\mathrm{s}}\right) .
$$

The Borel and Minimal Prescription differ in the way higher-twist behaviour of the resummed series is handled. However, in practice and as will be demonstrated in the next sections, this difference is negligible at collider energies we are interested in. The inclusion or exclusion of subleading terms in the Borel expression of eq. (2.20) is regulated by the cutoff $C$ which can be used to estimate the ambiguity of the resummation procedure. The larger the value of $C$ gets, the more we include power-correction terms in the series. However, as $C$ gets bigger the integral in eq. (2.20) becomes unstable and varies a lot for small changes of $C$ eventually spoiling the accuracy of the result. This is due to the fact that the series in eq. (2.12) is not Borel summable.

The following subsections deal with the numerical implementation of the procedure described above and, specifically, comment on the proper choice of the cutoff $C$ regulating the truncation of the Borel integral making it convergent. 


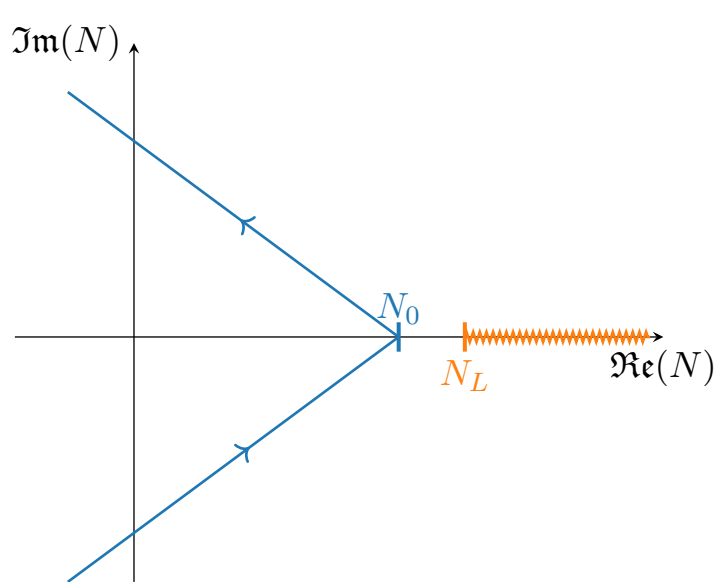

Figure 2. Singularity structures related to the Landau pole and the Minimal prescription path for the Mellin inverse integration.

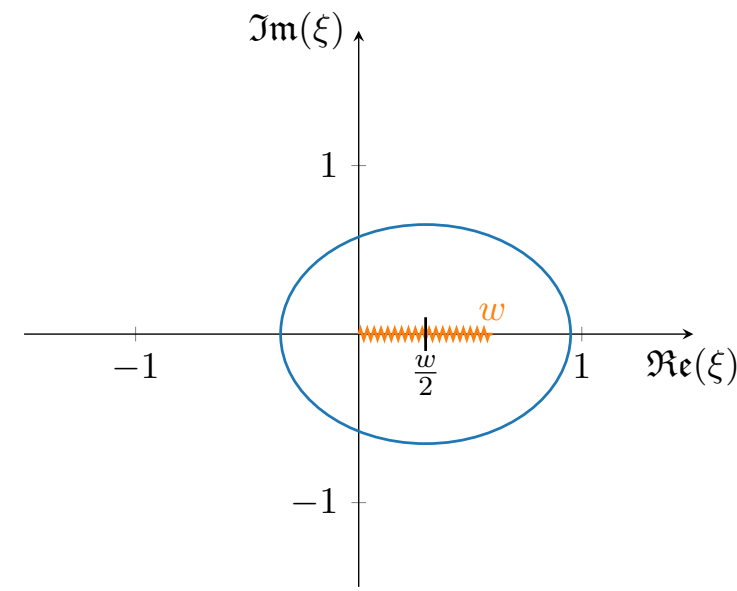

Figure 3. Branch cut of the integrand term $M\left(N, \xi_{p}, \xi\right) \Sigma\left(N, w / \xi, \alpha_{s}\right)$ in the $w$-integral given in eq. (2.20); $H$ is given by the blue circle.

\subsubsection{Contour and Borel integration}

By examining the singularity structure of the integrand in eq. (2.20) (see figure 3), we see that the contour integral can be written as an integral over the domain $[0,1]$,

$$
\frac{1}{2 \pi \mathrm{i}} \oint_{H} \mathrm{~d} \xi f(\xi)=R \int_{0}^{1} \mathrm{~d} \varphi \mathrm{e}^{2 \mathrm{i} \pi \varphi} f(\xi(\varphi)),
$$

where $f$ represents the integrand. The contour is parametrized by $\xi(\varphi)=x_{0}+R \exp (2 \mathrm{i} \pi \varphi)$ which is a circle around the real point $\xi=x_{0} \in \mathbb{R}$ with a radius $R>0$, in the positive direction around $x_{0}$. The branch cut and poles of $f$ are now mapped in terms of the variable $\xi$ into a branch cut on the real axis from zero to $w$ (see figure 3 ). In practice, we choose $x_{0}=w / 2$ and $R=w / 2+\zeta\left(\zeta \in \mathbb{R}^{*+}\right)$. The value of $\zeta$ and the cutoff $C$ must be chosen in such a way that the circle does not intersect the branch cut in $\xi$-space. Based on the twist- 4 argument [41], the value of the cutoff should be chosen according to $C \geq 2$. Throughout our implementation, we chose the minimal value for $C$ : i.e. $C=2$.

\subsubsection{Inverse Mellin}

Thanks to the Borel procedure, the Minimal Prescription can now be applied to perform the Mellin back transformation. The inverse Mellin transform given by eq. (2.10) can be rewritten in the following way

$$
\begin{aligned}
\frac{1}{2 \pi \mathrm{i}} \int_{N_{0}-\mathrm{i} \infty}^{N_{0}+\mathrm{i} \infty} \mathrm{d} N\left(\tau^{\prime}\right)^{-N} \frac{\mathrm{d} \sigma}{\mathrm{d} \xi_{p}}(N) & =\frac{1}{\pi} \operatorname{Im}\left[\int_{N_{0}}^{N_{0}+\mathrm{i} \infty} \mathrm{d} N\left(\tau^{\prime}\right)^{-N} \frac{\mathrm{d} \sigma}{\mathrm{d} \xi_{p}}(N)\right] \\
& =\frac{1}{\pi} \operatorname{Im}\left[(\mathrm{i}+r) \int_{0}^{1} \frac{\mathrm{d} u}{u}\left(\tau^{\prime}\right)^{-N(u)} \frac{\mathrm{d} \sigma}{\mathrm{d} \xi_{p}}(N(u))\right],
\end{aligned}
$$


where $N_{0}$, as usual, must be located to the right of all the singularities of the integrand. In the first line, we split the contour into two pieces, the first above the real axis and the second below. Since $\mathrm{d} \sigma / \mathrm{d} \xi_{p}\left(\tau^{*}\right)=\left[\mathrm{d} \sigma / \mathrm{d} \xi_{p}\left(\tau^{\prime}\right)\right]^{*}$ is real, the second piece turns out to be the negative of the complex conjugate of the first and hence can be combined to the former. In the second line, we used the substitution $N(u)=N_{0}+(r+\mathrm{i}) \ln u$ with $r>0$ being an arbitrary real and positive parameter which controls the slope of the path that enhances the numerical convergence of the integral. This parameter must be positive, so that for large imaginary values of $N$ (i.e. small values of $u$ ), the prefactor converges to zero,

$$
\left(\tau^{\prime}\right)^{-N(u)}=\exp \left(N(u) \ln 1 / \tau^{\prime}\right),
$$

requiring the real part of $N(u)$ to be negative for $u \rightarrow 0$ (i.e. $N_{0}-r \ln 1 / u<0$ ). Notice that the following is also an equivalent transformation:

$$
\frac{1}{2 \pi \mathrm{i}} \int_{N_{0}-\mathrm{i} \infty}^{N_{0}+\mathrm{i} \infty} \mathrm{d} N\left(\tau^{\prime}\right)^{-N} \frac{\mathrm{d} \sigma}{\mathrm{d} \xi_{p}}(N)=\frac{1}{\pi} \operatorname{Im}\left[(r-\mathrm{i}) \int_{0}^{1} \frac{\mathrm{d} u}{u}\left(\tau^{\prime}\right)^{-N(u)} \frac{\mathrm{d} \sigma}{\mathrm{d} \xi_{p}}(N(u))\right],
$$

where in this case $N(u)=N_{0}+(r-\mathrm{i}) \ln u$.

\subsection{PDF evolution for threshold-improved $p_{T}$ resummation}

In order to compute hadronic cross sections, we need to combine the partonic resummed cross section with the Parton Distribution Functions (PDFs) embodied in the parton luminosity $\mathcal{L}$ in eqs. (2.1) and (2.3). Transverse momentum resummation requires the evaluation of the PDFs at a scale which differs from the hard scale of the fixed-order calculation. The difference in scale is therefore taken care by PDF evolution functions. In the context of threshold-improved transverse momentum resummation, PDFs are evaluated at the scale $Q^{2} / \chi$ where $\chi$, as mentioned previously, depends both on the Mellin moment $N$ and the impact parameter $b$. Such a choice of scale, as a result, entails a new way of counting logarithms in the evolution which is the subject of this section.

The explicit expression of the partonic cross section $\mathrm{d} \hat{\sigma}_{a b}^{\mathrm{tr}} / \mathrm{d} \xi_{p}$ writes as:

$$
\begin{aligned}
\frac{\mathrm{d} \hat{\sigma}_{a b}^{\mathrm{tr}}}{\mathrm{d} \xi_{p}}\left(N, \chi, \alpha_{s}\right)= & \left(\sigma_{0}\right)_{c \bar{c}} \overline{\mathrm{H}}_{c}\left(\frac{\bar{N}^{2}}{\chi}, \alpha_{s}\left(Q^{2}\right)\right) C_{c i}\left(N, \alpha_{s}\left(\frac{Q^{2}}{\chi}\right)\right) C_{\bar{c} j}\left(N, \alpha_{s}\left(\frac{Q^{2}}{\chi}\right)\right) \\
& U_{i a}\left(N, \alpha_{s}\left(\frac{Q^{2}}{\chi}\right), \alpha_{s}\left(\mu_{F}^{2}\right)\right) U_{j b}\left(N, \alpha_{s}\left(\frac{Q^{2}}{\chi}\right), \alpha_{s}\left(\mu_{F}^{2}\right)\right) \exp \left(S_{c}\left(N, \chi, \mu_{R}^{2}\right)\right),
\end{aligned}
$$

where here $\overline{\mathrm{H}}$ represents the hard factor which behaves as a constant in the large $-\hat{b}$ limit, $C$ is the coefficient functions, $U$ is the evolution operators that evolve the PDFs from the scale $Q^{2} / \chi$ to $\mu_{F}$, and finally, $S_{c}$ is the Sudakov form factor. The subscripts define partonic indices where repeated indices are summed over. In the case of the Higgs production via gluon fusion, for instance, $c=g$. Finally, $\mu_{R}$ and $\mu_{F}$ represent the renormalization and factorization scale respectively. From eq. (2.25), we can see that the modified resummed expression differs from the standard $p_{T}$ resummation in two ways. First, the argument 
of the logarithms in the Sudakov exponent includes the soft contribution. Second, as mentioned above, the argument of the running of coupling, both in the coefficient functions and in the evolution operators, is computed at the scale $Q^{2} / \chi$ instead of $b_{0}^{2} / b^{2}$.

In order to determine the appropriate logarithmic counting, we work out in this section the solution of the evolution equation explicitly. Let us first start with the coefficient functions $C$ of eq. (2.25). One can set all the arguments of $\alpha_{s}$ in $C$ to be the same and take into account the difference in scales in an evolution factor $R$ such that

$$
C_{c i}\left(N, \alpha_{s}\left(\frac{Q^{2}}{\chi}\right)\right)=C_{c i}\left(N, \alpha_{s}\left(Q^{2}\right)\right) R_{c i}\left(N, \alpha_{s}\left(\frac{Q^{2}}{\chi}\right), \alpha_{s}\left(Q^{2}\right)\right)
$$

where

$$
R_{c i}\left(N, \alpha_{s}\left(\frac{Q^{2}}{\chi}\right), \alpha_{s}\left(Q^{2}\right)\right)=\exp \left\{-\int_{Q^{2} / \chi}^{Q^{2}} \frac{\mathrm{d} q^{2}}{q^{2}} \frac{\beta\left(\alpha_{s}\left(q^{2}\right)\right)}{\alpha_{s}\left(q^{2}\right)}\left[\frac{\mathrm{d} \ln C_{c i}\left(N, \alpha_{s}\left(q^{2}\right)\right)}{\mathrm{d} \ln \alpha_{s}\left(q^{2}\right)}\right]\right\} .
$$

Let us now turn to the PDF evolution. Here, we can evolve the PDFs from $Q^{2} / \chi$ to $Q^{2}$ and then from $Q^{2}$ to $\mu_{F}^{2}$. In the case where $Q=\mu_{F}$, we have $U_{i j}\left(N, \alpha_{s}\left(Q^{2}\right), \alpha_{s}\left(\mu_{F}^{2}\right)\right)=1$. The expression of the PDF evolution can be written as [42]:

$$
\begin{aligned}
U_{i a}\left(N, \alpha_{s}\left(\frac{Q^{2}}{\chi}\right), \alpha_{s}\left(Q^{2}\right)\right)= & V_{i l}\left(N, \alpha_{s}\left(\frac{Q^{2}}{\chi}\right)\right) U_{l k}^{(\mathrm{LO})}\left(N, \alpha_{s}\left(\frac{Q^{2}}{\chi}\right), \alpha_{s}\left(Q^{2}\right)\right) \\
& \tilde{V}_{k a}\left(N, \alpha_{s}\left(Q^{2}\right)\right),
\end{aligned}
$$

where $U^{(\mathrm{LO})}$ and $V$ respectively represent the lowest and higher perturbative order solutions to the evolution equation, and $\tilde{V}_{k a}$ denotes the $(k, a)$-element of the inverse matrix $\mathbf{V}^{-1}$ in the flavour space. Henceforth, we shall use the boldface notation to denote the representation in flavour matrix space. In the case of singlet, the lowest perturbative order solution is derived by diagonalizing the LO anomalous dimensions matrix $\gamma^{(0)}$. Thus, following refs. [29, 42], we have

$$
U_{l k}^{(\mathrm{LO})}\left(N, \alpha_{s}\left(\frac{Q^{2}}{\chi}\right), \alpha_{s}\left(Q^{2}\right)\right)=\sum_{r= \pm} e_{l k}^{(r)}(N) \exp \left\{-\frac{\lambda_{r}^{(0)}(N)}{\beta_{0}} \ln \left(\frac{\alpha_{s}\left(Q^{2} / \chi\right)}{\alpha_{s}\left(Q^{2}\right)}\right)\right\},
$$

where $\lambda_{ \pm}$and $e^{ \pm}$represent the eigenvalues of the singlet matrix $\gamma^{(0)}$ and its projectors respectively whose expressions are given by eqs. (2.27) and (2.28) of [42]. On the other hand, the functions $V$ which take into account the higher order solutions to the evolution equation can be perturbatively expanded as a series in $\alpha_{s}$,

$$
V_{i l}(N, \mu)=\delta_{i l}+\sum_{n=1}^{\infty} \alpha_{s}^{(n)}(\mu) V_{i l}^{(n)}(N) .
$$

Each coefficient $V^{(i)}$ in eq. (2.30) can be computed iteratively following [42]. For instance, the first order coefficient $\mathbf{V}^{(1)}$ is given by

$$
\mathbf{V}^{(1)}(N)=\sum_{i, j= \pm} \frac{1}{\lambda_{j}^{(0)}(N)-\lambda_{i}^{(0)}(N)-\beta_{0}} \mathbf{e}^{(i)}(N)\left(\gamma^{(1)}(N)-\frac{\beta_{1}}{\beta_{0}} \gamma^{(0)}(N)\right) \mathbf{e}^{(i)}(N),
$$

where we have introduced the coefficients $\beta_{i}$ of the QCD $\beta$-function. 
Once again, one can take into account the difference in the argument of the running of coupling of $V\left(N, \alpha_{s}\left(Q^{2} / \chi\right)\right)$ in eq. (2.28a) by introducing an evolution factor

$$
V_{i l}\left(N, \alpha_{s}\left(\frac{Q^{2}}{\chi}\right)\right)=V_{i l}\left(N, \alpha_{s}\left(Q^{2}\right)\right) E_{i l}\left(N, \alpha_{s}\left(\frac{Q^{2}}{\chi}\right), \alpha_{s}\left(Q^{2}\right)\right)
$$

where similarly to eq. (2.26),

$$
E_{i l}\left(N, \alpha_{s}\left(\frac{Q^{2}}{\chi}\right), \alpha_{s}\left(Q^{2}\right)\right)=\exp \left\{-\int_{Q^{2} / \chi}^{Q^{2}} \frac{\mathrm{d} q^{2}}{q^{2}} \frac{\beta\left(\alpha_{s}\left(q^{2}\right)\right)}{\alpha_{s}\left(q^{2}\right)}\left[\frac{\mathrm{d} \ln V_{i l}\left(N, \alpha_{s}\left(q^{2}\right)\right)}{\mathrm{d} \ln \alpha_{s}\left(q^{2}\right)}\right]\right\} .
$$

At this point, we can discuss the difference in counting between threshold-improved and standard $p_{T}$ resummation. In the context of standard transverse momentum resummation, the leading-order solution to the evolution equation contributes with a single logarithm of $\hat{b}$. However, from the point of view of the threshold resummation, the flavour diagonal anomalous dimensions carry an additional contribution of the form $A_{g / q}^{p_{T}} \ln \left(\bar{N}^{2}\right)$. To see this, let us compute the term in the exponent of the lowest-order solution to the evolution equation given by eq. (2.29):

$$
\mathrm{G}_{ \pm}\left(N, \chi, \alpha_{s}\right) \equiv-\frac{\lambda_{ \pm}^{(0)}(N)}{\beta_{0}} \ln \left(\frac{\alpha_{s}\left(Q^{2} / \chi\right)}{\alpha_{s}\left(Q^{2}\right)}\right)=\frac{\gamma_{g g / q q}^{(0)}}{\beta_{0}} \ln \left(1-\lambda_{\chi}\right)+\mathcal{O}\left(\alpha_{s}\left(Q^{2}\right)\right) .
$$

Taking the large- $N$ limit of the anomalous dimension leads to the following result

$$
\frac{\gamma_{g g / q q}^{(0)}}{\beta_{0}} \ln \left(1-\lambda_{\chi}\right) \stackrel{N \rightarrow \infty}{\longrightarrow}-\frac{A_{g / q}^{p_{T}}}{\beta_{0}} \ln (\bar{N}) \ln \left(1-\lambda_{\chi}\right)=-\frac{A_{g / q}^{p_{T}}}{2 \bar{\alpha}_{s} \beta_{0}} \lambda_{N} \ln \left(1-\lambda_{\chi}\right),
$$

where we have defined $\lambda_{N}=\bar{\alpha}_{s} \ln \left(\bar{N}^{2}\right)$. Therefore, in order to compute $\mathrm{N}^{k}$ LL thresholdimproved $p_{T}$ resummation, the large- $N$ behaviour of the evolution has to be included up to $\mathrm{N}^{k} \mathrm{LO}$, which is not the case in the standard transverse momentum resummation as $\mathrm{N}^{k-1} \mathrm{LO}$ is enough. That is, at NNLL, the solution of evolution is performed up to NNLO accuracy but with the NNLO anomalous dimension substituted by its large- $N$ behaviour.

Combining all the results, we can organize terms in such a way that we obtain the form presented in eq. (2.9). The perturbative function $\tilde{\mathcal{H}}=\sum_{n=0}^{\infty} \alpha_{s}^{n} \tilde{\mathcal{H}}^{(n)}$ is then given by

$$
\begin{aligned}
\tilde{\mathcal{H}}_{a b}^{\{\mathcal{P}\}}(N, \chi)=\overline{\mathrm{H}}_{c}\left(\frac{\bar{N}^{2}}{\chi}, \alpha_{s}\left(Q^{2}\right)\right) \tilde{C}_{c l}\left(N, \alpha_{s}\left(Q^{2}\right)\right) \tilde{C}_{\bar{c} m}\left(N, \alpha_{s}\left(Q^{2}\right)\right) \\
e_{l k}^{(r)}(N) \tilde{V}_{k a}\left(N, \alpha_{s}\left(Q^{2}\right)\right) e_{m n}^{(p)}(N) \tilde{V}_{n b}\left(N, \alpha_{s}\left(Q^{2}\right)\right)
\end{aligned}
$$

where here $\{\mathcal{P}\}=\{r, p\}= \pm$ and we have defined $\tilde{C}_{c l}=C_{c i} V_{i l}$. Finally, the universal form factor in the exponent of eq. (2.9) which contains all the logarithmic enhanced terms is expressed as

$$
\mathcal{G}^{\{\mathcal{P}\}}\left(N, \lambda_{\chi}\right)=S_{c}+\mathrm{G}_{r}+\mathrm{G}_{p}+\ln \left(\tilde{R}_{c l} \tilde{R}_{c m}\right)
$$

where the evolution factor $\tilde{R}_{c l}$ is defined as $\tilde{R}_{c l}=R_{c i} E_{i l}$. The logarithmic expansion of $S_{c}$ starts at LL accuracy. While the expansion of $\mathrm{G}_{r / p}$ starts at NLL accuracy in the 
standard $p_{T}$ resummation, their large- $N$ behaviour already contribute at LL in the context of modified $p_{T}$ resummation in order to take into account for the soft behaviour. Similarly to the standard $p_{T}$ resummation, the flavour off-diagonal terms of $\tilde{R}_{c l}(c \neq l)$ starts to contribute at NLL. The terms in the flavour diagonal, instead, start to contribute at NLL as opposed to the standard resummation procedure where they start to contribute at NNLL.

To summarize, the truncation of the threshold-improved transverse momentum resummation at a given logarithmic accuracy is defined in the following way:

- At LL, we approximate the hard function $\tilde{\mathcal{H}}$ by its lowest perturbative order and include $g_{1}$ in the Sudakov exponent. The $\mathrm{G}_{ \pm}$functions are included up to LO with $\gamma^{(0)}$ replaced by its large- $N$ behaviour while the $\tilde{R}$ functions are approximated to 1 .

- At NLL, we include $\tilde{\mathcal{H}}^{(1)}$ in $\tilde{\mathcal{H}}$ with the function $g_{2}$ in the Sudakov exponent. In addition, we include the complete LO term in $\mathrm{G}_{ \pm}$together with the NLO term where $\gamma^{(0)}$ and $\gamma^{(1)}$ are replaced the their large- $N$ behaviour. The full expression of $\tilde{R}_{g q}$ is also included while we only include the large- $N$ behaviour of $V^{(1)}(N)$ in $\tilde{R}_{g g}$.

- At NNLL, we include in $\tilde{\mathcal{H}}$ the coefficient $\tilde{\mathcal{H}}^{(2)}$ and the function $g_{3}$ in the Sudakov exponent. Similarly, we also include the complete NLO term in $\mathrm{G}_{ \pm}$together with the NNLO term where the anomalous dimensions are replaced by their large- $N$ behaviour. Finally, we add to $\tilde{R}_{g g}$ the large- $N$ behaviour of $V^{(2)}(N)$.

\subsection{Finite order truncation of the resummed expression}

In order to provide a valid prediction up to NNLL+NLO, we need to match the resummed results to the fixed-order calculation. This matching procedure guarantees the correct behaviour from perturbative calculations up to a specified order and incorporates the large logarithms from resummation at higher-orders. The matching consists on adding to the allorder results the perturbative calculations truncated at a given order in $\alpha_{s}$ and subtract to the whole the expansion of the resummed results at the same order. The matched expression of the combined cross section is therefore given by

$$
\frac{\mathrm{d} \hat{\sigma}_{a b}^{\mathrm{match}}}{\mathrm{d} \xi_{p}}=\frac{\mathrm{d} \hat{\sigma}_{a b}^{\mathrm{resum}}}{\mathrm{d} \xi_{p}}+\left[\frac{\mathrm{d} \hat{\sigma}_{a b}^{\mathrm{F}} \mathrm{O}}{\mathrm{d} \xi_{p}}\right]_{\mathcal{O}\left(\alpha_{s}^{n}\right)}-\left[\frac{\mathrm{d} \hat{\sigma}_{a b}^{\exp }}{\mathrm{d} \xi_{p}}\right]_{\mathcal{O}\left(\alpha_{s}^{n}\right)} .
$$

The subscripts $\mathcal{O}\left(\alpha_{s}^{n}\right)$ indicate the order at which both the fixed-order and the expanded expressions are truncated. Here, the notation used to denote the fixed-order (F.O) accuracy and therefore the matched result refers to the accuracy of the $p_{T}$ distribution. In particular, the LO refers to the non-trivial order of the transverse momentum distribution whose integral is the NLO total cross section. In the sequel, LO and NLO will always refer to the accuracy of the $p_{T}$ spectrum. The expansion of $\Sigma_{a b}$ will give rise to a series of the form

$$
\Sigma_{a b}^{\exp }\left(N, \chi, \alpha_{s}\right)=\left(\sigma_{0}\right)_{c \bar{c}}\left\{\delta_{c a} \delta_{\bar{c} b}+\sum_{n=1}^{\infty} \alpha_{s}^{n}\left[\Sigma_{a b}^{(n)}(N, \chi)+\mathcal{H}_{a b}^{(n)}(N, \chi)\right]\right\} .
$$

The perturbative coefficients $\Sigma^{(n)}$ are polynomials in the logarithm variable $\ln (\chi)$. They vanish when $\ln (\chi)=0$ (i.e. $\hat{b}=-\bar{N}^{2} b_{0}^{2}$ ) and lead to threshold resummation when $b=0$. 
On the other hand, the function $\mathcal{H}^{(n)}$ contains all the terms that behave as constants in the large- $\hat{b}$ limit. The explicit expressions of the two perturbative coefficient functions $\Sigma^{(1)}$ and $\Sigma^{(2)}$ which contribute in eq. (2.39) are given by

$$
\begin{aligned}
\Sigma_{a b}^{(1)}(N, \chi)= & \Sigma_{a b}^{(1 ; 2)}(N) \ln ^{2}(\chi)+\Sigma_{a b}^{(1 ; 1)}(N) \ln (\chi), \\
\Sigma_{a b}^{(2)}(N, \chi)= & \Sigma_{a b}^{(2 ; 4)}(N) \ln ^{4}(\chi)+\Sigma_{a b}^{(2 ; 3)}(N) \ln ^{3}(\chi)+ \\
& \Sigma_{a b}^{(2 ; 2)}(N) \ln ^{2}(\chi)+\Sigma_{a b}^{(2 ; 1)}(N) \ln (\chi) .
\end{aligned}
$$

The functions $\Sigma^{(i ; j)}(N)$ are purely functions of $N$. In order to match eq. (2.39) to the fixedorder results, we need to Fourier-invert logarithms of the form $\ln ^{k}(\chi)$, such computation requires the evaluation of the following integral at a given order in $k$

$$
\Im_{k}\left(N, \xi_{p}\right)=\int_{0}^{\infty} \mathrm{d} \hat{b} \frac{\hat{b}}{2} J_{0}\left(\hat{b} \sqrt{\xi_{p}}\right) \ln ^{k}\left(\bar{N}^{2}+\frac{\hat{b}^{2}}{b_{0}^{2}}\right) .
$$

Thus, according to eq. (2.15), the computation of $\mathfrak{I}_{k}$ for a fixed value of $k$ just amounts to the computation of the following limit

$$
\mathfrak{I}_{k}\left(N, \xi_{p}\right)=\lim _{\epsilon \rightarrow 0} \frac{\partial^{k}}{\partial \epsilon^{k}} M\left(N, \xi_{p}, \epsilon\right),
$$

with $M$ expressed as in eq. (2.15). This allows us to analytically compute the inverse Fourier of the finite order expansion of the resummed expression up to $k=4$,

$$
\begin{aligned}
\mathfrak{I}_{1}\left(N, \xi_{p}\right)= & -\frac{2 N}{\sqrt{\xi_{\mathrm{p}}}} \mathrm{K}_{1}(2 N \sqrt{\xi \mathrm{p}}) \\
\mathfrak{I}_{2}\left(N, \xi_{p}\right)= & -\frac{4 N}{\sqrt{\xi_{\mathrm{p}}}} \mathrm{K}_{1}^{(1)}(2 N \sqrt{\xi \mathrm{p}})-\frac{4 N}{\sqrt{\xi_{\mathrm{p}}}}\left(\ln \left(\frac{N}{\sqrt{\xi_{\mathrm{p}}}}\right)+\gamma_{E}\right) \mathrm{K}_{1}\left(2 N \sqrt{\xi_{\mathrm{p}}}\right) \\
\mathfrak{I}_{3}\left(N, \xi_{p}\right)= & -\frac{6 N}{\sqrt{\xi_{\mathrm{p}}}} \mathrm{K}_{1}^{(2)}\left(2 N \sqrt{\xi_{\mathrm{p}}}\right)-\frac{12 N}{\sqrt{\xi_{\mathrm{p}}}}\left(\ln \left(\frac{N}{\sqrt{\xi_{\mathrm{p}}}}\right)+\gamma_{E}\right) \mathrm{K}_{1}^{(1)}\left(2 N \sqrt{\xi_{\mathrm{p}}}\right) \\
& -\frac{6 N}{\sqrt{\xi_{\mathrm{p}}}}\left(\ln ^{2}\left(\frac{N}{\sqrt{\xi_{\mathrm{p}}}}\right)+2 \gamma_{E} \ln \left(\frac{N}{\sqrt{\xi_{\mathrm{p}}}}\right)-\zeta_{2}+\gamma_{E}^{2}\right) \mathrm{K}_{1}\left(2 N \sqrt{\xi_{\mathrm{p}}}\right) \\
\mathfrak{I}_{4}\left(N, \xi_{p}\right)= & -\frac{8 N}{\sqrt{\xi_{\mathrm{p}}}} \mathrm{K}_{1}^{(3)}(2 N \sqrt{\xi \mathrm{p}})-\frac{24 N}{\sqrt{\xi_{\mathrm{p}}}}\left(\ln { }^{2}\left(\frac{N}{\sqrt{\xi_{\mathrm{p}}}}\right)+2 \gamma_{E} \ln \left(\frac{N}{\sqrt{\xi_{\mathrm{p}}}}\right)-\zeta_{2}+\gamma_{E}^{2}\right) \\
& \mathrm{K}_{1}^{(1)}\left(2 N \sqrt{\xi_{\mathrm{p}}}\right)-\frac{24 N}{\sqrt{\xi_{\mathrm{p}}}}\left(\ln \left(\frac{N}{\sqrt{\xi_{\mathrm{p}}}}\right)+\gamma_{E}\right) \mathrm{K}_{1}^{(2)}\left(2 N \sqrt{\xi_{\mathrm{p}}}\right)-\frac{8 N}{\sqrt{\xi_{\mathrm{p}}}} \mathrm{K}_{1}\left(2 N \sqrt{\xi_{\mathrm{p}}}\right) \\
& \left(\ln ^{3}\left(\frac{N}{\sqrt{\xi_{\mathrm{p}}}}\right)+3 \gamma_{E} \ln ^{2}\left(\frac{N}{\sqrt{\xi_{\mathrm{p}}}}\right)+3\left(\gamma_{E}^{2}-\zeta_{2}\right) \ln \left(\frac{N}{\sqrt{\xi_{\mathrm{p}}}}\right)-3 \gamma_{E} \zeta_{2}+\gamma_{E}^{3}+\zeta_{3}\right),
\end{aligned}
$$

where the $\mathrm{K}_{1}^{(i)}$ are the $i$-th derivatives of the Bessel function w.r.t. the argument. One can check that in the small- $p_{T}$ limit - meaning taking $N \rightarrow \exp \left(-\gamma_{E}\right)$ - the above expressions exactly reproduce the standard small- $p_{T}$ expressions given by eqs. (121)-(124) 
of ref. [29]. The equivalence between taking $p_{T} \rightarrow 0$ and setting $N \rightarrow \exp \left(-\gamma_{E}\right)$ deserves further comment. On can check that the modified logarithmic variable $\ln \left(1+\hat{b}^{2} / b_{0}^{2}\right)$ of ref. [29] is obtained from our modified $\ln (\chi(\hat{b}))$ by setting $\bar{N}=1$ which in eqs. (2.43) translates into taking $N \rightarrow \exp \left(-\gamma_{E}\right)$.

\section{Phenomenological studies}

In the previous sections, we have developed a framework that allows us to numerically perform the inverse Fourier-Mellin transform of the resummed expression and to match it to the fixed-order result. We now turn to the main result of this paper, namely a detailed phenomenological study of the impact of the threshold-improved transverse momentum and combined resummation on transverse momentum distributions in the case of Higgs and Z-boson production. Our aim is to assess the potential impact of: (i) the improved transverse momentum resummation through the inclusion of the soft contributions which we expect to be more visible in the small- $p_{T}$ region, and (ii) the soft resummation at fixed- $p_{T}$ which we expect to improve the matching to fixed-order in the intermediate and large- $p_{T}$ regions.

All results are produced using the NNPDF31_nnlo_as_0118 set of parton distributions from NNPDF3.1 [43] through the LHAPDF [44] interface in the manner described in refs. $[32,41]$ by expanding the parton luminosity in the basis of the Chebyshev polynomials; we expect the results to be largely independent of the PDF set used. In order to assess perturbative uncertainties and higher order corrections, we perform standard variations of factorization and renormalization scales using the seven-point method. Notice, however, that the present studies do not include resummation scale variation as in ref. [29], which will be done in future studies at $\mathrm{N}^{3} \mathrm{LL}$. In addition to defining a proper value to the cutoff $C$ entering in the Borel prescription, one also has to define the free parameters entering the matching function in eq. (2.6). The following results are produced by setting $k=3$ and $m=2$. There is some arbitrariness in the definition of $k$ and $m$; however, we did explicitly check that results do not change provided that $k$ is chosen from 2 to 5 and $m<k$. The resummed results are produced from our own resummation code while the fixed-order part is provided by the NNLOJET [45] implementation.

\subsection{Threshold-improved $p_{T}$ resummation in the large- $\hat{b}$ limit}

In this section, we start by checking that our results reproduce the standard transverse momentum resummation when the soft contributions are switched off. Besides being a consistency check, this allows us to verify that the Borel prescription agrees with the Minimal Prescription (MP) of ref. [29]. It has been shown analytically in ref. [16] that thresholdimproved $p_{T}$ resummation produces exactly standard $p_{T}$ resummation in the large- $\hat{b}$ limit, here, we show numerically that this is indeed the case using the Borel prescription. Taking $\hat{b} \rightarrow \infty$ means that we adopt the following replacements throughout our expressions:

$$
\chi=\bar{N}^{2}+\frac{\hat{b}^{2}}{b_{0}^{2}} \longrightarrow 1+\frac{\hat{b}^{2}}{b_{0}^{2}}, \quad \text { and } \quad \frac{Q}{\chi} \longrightarrow \frac{b_{0}^{2}}{b^{2}} .
$$



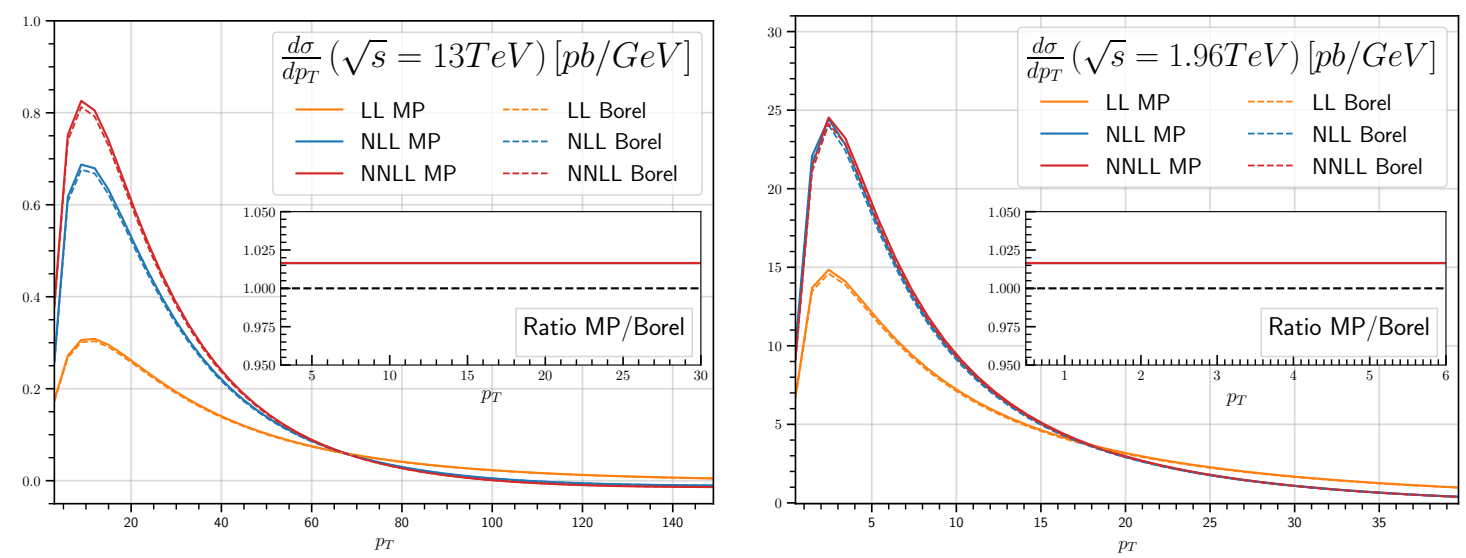

Figure 4. Comparison of the Minimal and Borel prescription for the transverse momentum distribution of the Higgs (left) and $Z$ boson (right). The renormalization $\left(\mu_{R}\right)$ and factorization $\left(\mu_{F}\right)$ scales are set equal to the Higgs mass $\left(m_{H}\right)$ and the $Z$ boson mass $\left(m_{Z}\right)$ in the case of Higgs and DY production respectively. The normalizations are fixed to the total cross section.

Notice that in the expression of $\chi, \bar{N}$ has been replaced by 1. As suggested in ref. [29], shifting the argument of the logarithm by 1 reduces the effect of unjustified small- $p_{T}$ resummation in the large- $p_{T}$ region (equivalently $\hat{b} \rightarrow 0$ ). The replacements in eq. (3.1) implies that we have to change the function $M$ in the following way,

$$
\tilde{M}\left(\xi_{p}, \epsilon\right)=2 \mathrm{e}^{2 \gamma_{E} \epsilon}\left(\frac{1}{\sqrt{\xi_{p}}}\right)^{1+\epsilon} \frac{K_{1+\epsilon}\left(2 \sqrt{\xi_{p}}\right)}{\Gamma(-\epsilon)} .
$$

In order to check the consistency of the Borel formalism, we perform the replacement in eqs. (3.1) and (3.2), and we implement the exact same procedure as in ref. [29] by suitably adjusting the solutions to the evolution equation and fixing the integral over $p_{T}$ to be the fixed-order total cross section. The results shown in figure 4 demonstrates that our formalism is order by order consistent with the standard small- $p_{T}$ results from HqT [29] for Higgs and DYqT $[36,46]$ for DY which both use the Minimal Prescription. We note that apart from the changes mentioned above, no further treatment is needed for the Borel approach as the two formalisms only differ in the way the asymptotic nature of the resummed series is treated. This confirms two things: first, that the threshold-improved $p_{T}$ resummation indeed reproduces the standard small- $p_{T}$ resummation in the correct limit; and second, that the Borel method is a meaningful prescription to perform the inverse Fourier-Mellin transform.

\subsection{Higgs boson production at LHC and DY production at Tevatron}

We begin by studying the standard model Higgs produced via gluon-gluon fusion at LHC with a center of mass energy of $\sqrt{s}=13 \mathrm{TeV}$. The computations have been performed in the large-top-mass $\left(m_{T}\right)$ limit with a Higgs mass $m_{H}=125 \mathrm{GeV}$. As mentioned previously, the factorization and renormalization scales are varied according to the seven-point method by a factor of two in either direction around $m_{H}$. 
The left plots of figure 5 study the impact of the threshold improved small- $p_{T}$ (TIpT, henceforth) (figure 5c) and combined (figure 5e) resummation on the transverse momentum distribution by comparing the results to the NLO results. The standard small- $p_{T}$ (SSpT, henceforth) resummation is shown in figure 5a. The top panels show the different order of resummation (NLL in blue and NNLL in red) along with the NLO results (orange). The ratio w.r.t. the NNLL result is shown in the lower panels for the different types of resummation (SSpT, TIpT, and combined). In contrast to the NLO fixed-order results, the resummation leads to a well-behaved transverse momentum distribution that has peak at $p_{T} \sim 10 \mathrm{GeV}$ and vanishes for $p_{T} \rightarrow 0$. As expected, we observe that the scale uncertainty is reduced with the inclusion of the NNLL terms. This feature is seen in all three types of resummations, but it is more pronounced in the small- $p_{T}$ region. Threshold-improved and standard $p_{T}$ resummation agree in the intermediate and large- $p_{T}$ region at NLL, but a sizeable difference appears in the small- $p_{T}$ range (below $\sim 25 \mathrm{GeV}$ ). Indeed, we see that the threshold-improved transverse momentum resummation displays faster convergence at small values of $p_{T}$. As a consequence, in threshold-improved $p_{T}$ resummation, the NLL result is much closer to the NNLL result than in SSpT resummation. However, at NNLL, the difference between SSpT and TIpT resummation is almost invisible, but with the thresholdimproved $p_{T}$ resummation having a moderately smaller uncertainty. Turning now to the combined result, threshold-improved $p_{T}$ and combined resummation are exactly similar up to scales of at least $40 \mathrm{GeV}$ where the pure threshold resummation starts to contribute. The inclusion of the threshold resummation leads to a surprising agreement between of the NNLL resummed result and the fixed-order, although a noticeable difference persists between the NLL and NLO results for larger values of $p_{T}$. Perhaps the most interesting feature of the combined resummation is the fact that even in the absence of matching the resummed computation seems to capture the behaviour of the fixed-order in the region where standard $p_{T}$ resummation fails to give accurate predictions. As a side note, unlike standard $p_{T}$ resummation, our modified resummation does not require any regulation procedure to get rid of its large- $p_{T}$ behaviour. Instead, it relies on the fact that its $\hat{b}=0$ limit coincides with the threshold resummed inclusive cross section that fixes the integral of the distribution.

Next, in order to fully obtain the most accurate predictions, we match the resummed expressions to the fixed-order results according to eq. (2.38). The results are presented on the right-hand side of figure 5. The upper panels show the comparison between NLL+LO (blue), NNLL+NLO (red), and NLO (orange) for the three types of resummations. The lower panels show the ratio w.r.t. the central scale (i.e. $\mu_{R}=\mu_{F}=m_{H}$ ) of NNLL+NLO. By comparing figure $5 \mathrm{~b}$ with figure $5 \mathrm{~d}$, we see faster convergence for the threshold-improved $p_{T}$ resummation. However, we notice that in the absence of the additional threshold resummed expression, the NLL+LO threshold-improved $p_{T}$ resummation underestimates corrections from missing higher orders. This can be seen in figure $5 \mathrm{~d}$ where the NLL+LO band shifts away from both the NLO and NNLL+NLO results. At small $p_{T}$, threshold-improved $p_{T}$ and combined resummation are exactly similar. The difference only occurs in the mediumrange- $p_{T}$ where the contribution from the soft resummation again improves the agreement of the combined with the fixed-order result. In particular, we would like to highlight the good agreement between the resummed and the fixed-order results at large values of $p_{T}$. 

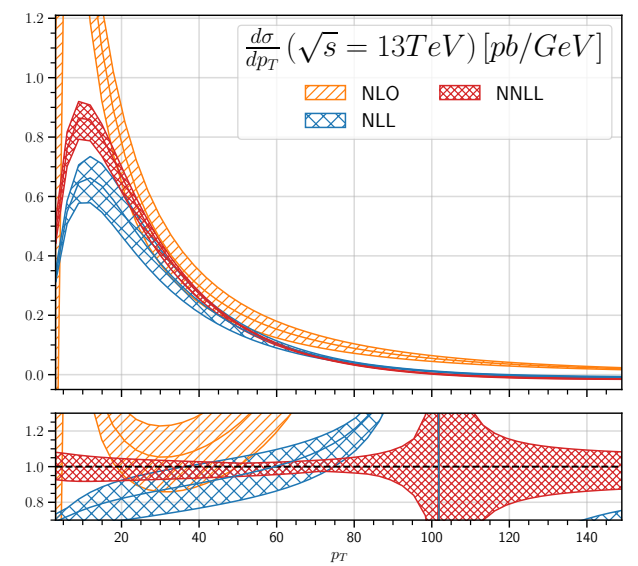

(a) SSpT resummation
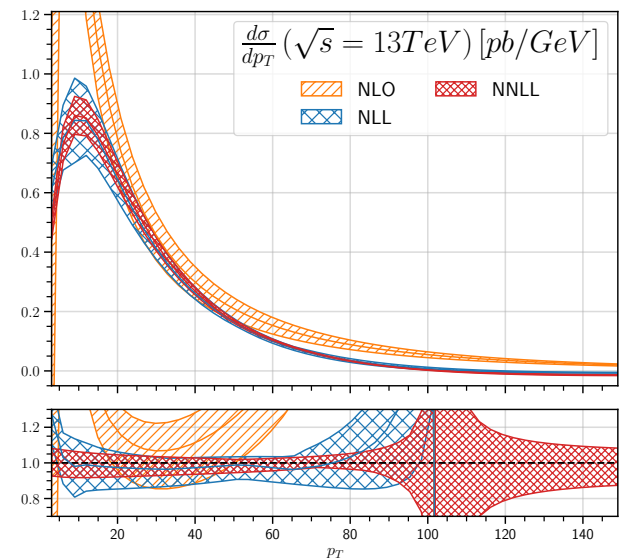

(c) TIpT resummation
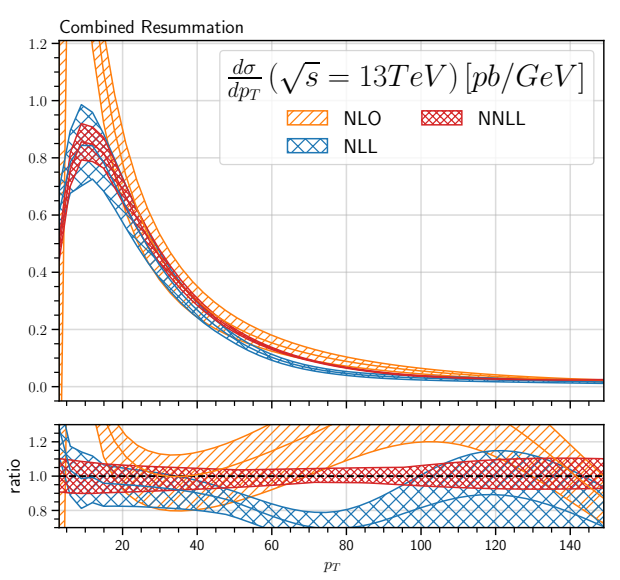

(e) combined resummation
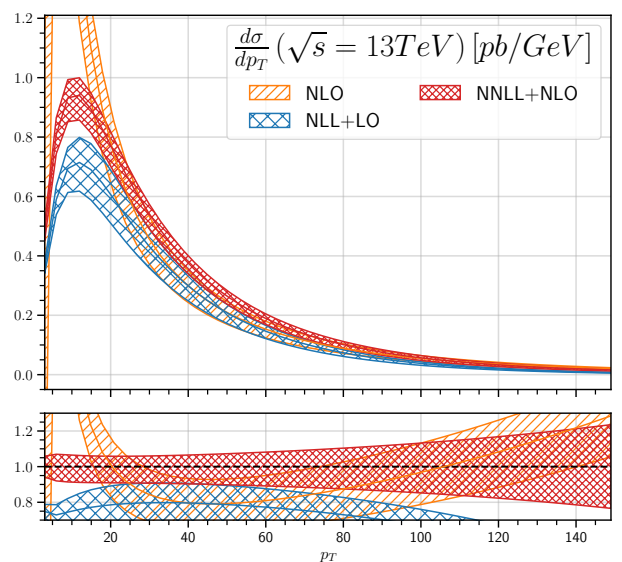

(b) SSpT resummation with matching
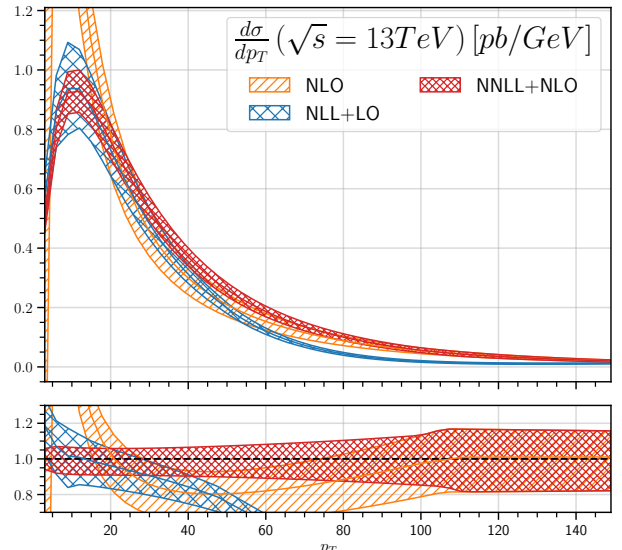

(d) TIpT resummation with matching
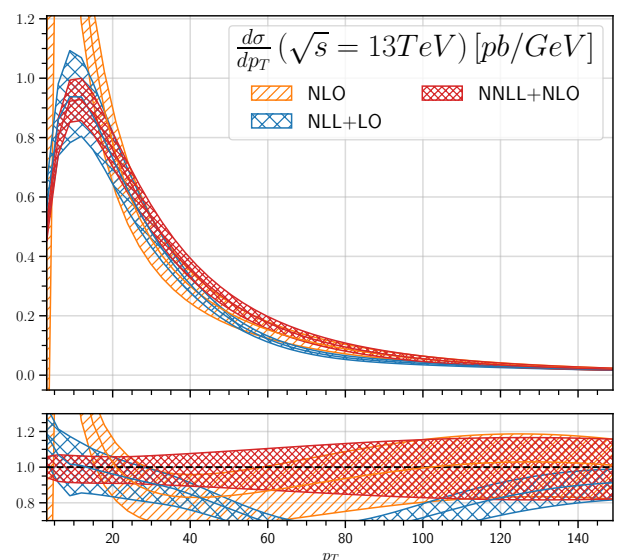

(f) combined resummation with matching.

Figure 5. $p_{T}$ spectrum of the Higgs boson production for various types of resummation: standard small- $p_{T}$ (top), threshold-improved small- $p_{T}$ (middle), and combined (bottom). The left plots show the pure resummation results while those matched to the fixed-order are shown on the right. The top panels compare the NLL and NNLL resummed results with the NLO. The lower panels show the ratio w.r.t. to the central value of the respective NNLL results. The uncertainty bands are computed by varying $\mu_{R}$ and $\mu_{F}$ using the 7-point method; in all cases, $Q=m_{H}$. 
In the context of threshold-improved $p_{T}$ and combined results, the matched results have broadly similar features to the purely resummed ones. The main new feature is that the matching of the combined resummation changes the result less than the matching of the TIpT resummation, and the latter less than the matching of the SSpT resummation.

We now turn to the application of the threshold-improved transverse momentum and combined resummation to a $Z$-boson production at the Tevatron via DY mechanism. We consider the production of a $Z$ boson with mass $m_{Z}=91.187 \mathrm{GeV}$ at the Tevatron with a center of mass energy $\sqrt{s}=1.96 \mathrm{TeV}$ because soft contributions for DY processes are expected to be more pronounced at colliders with lower center of mass energy.

Figure 6 shows the results in comparison to the standard $p_{T}$ resummation. Similar to Higgs results in figure 5, the pure resummed results are shown on the left-hand side. In all three types of resummations, one sees similar features as previously, namely the fact that the distributions go to zero as $p_{T}$ gets very small. The distributions then peak at about $p_{T} \sim 2 \mathrm{GeV}$ before vanishing again at large $p_{T}$. Both at NLL and NNLL, threshold-improved $p_{T}$ resummation (figure $6 \mathrm{c}$ ) is analogous to standard transverse momentum (figure 6a) at low $p_{T}$; small but yet noticeable differences occur at large $p_{T}$ values, where the modified $p_{T}$ resummation displays slightly better convergence. This feature is more accentuated in the combined resummation (figure 6e) yielding a good agreement with the NLO (orange) results. Again, these results suggest the validity of the thresholdimproved $p_{T}$ and combined resummation even at large values of $p_{T}$ where the use of only standard $p_{T}$ resummation is not fully justified.

This behaviour remains when we match the pure resummed calculation to the fixedorder. The results are shown on the right-hand side of figure 6. In particular, we can notice the good agreement between the NLL+LO, NNLL+NLO, and NLO results in the combined case (figure 6f). Not only is the NNLL+NLO band smaller compared to the NLL+LO, but the latter is also contained in the former suggesting a good convergence of the resummed perturbative expansion. These results suggest that for the transverse momentum distribution of the $Z$ boson where $x$ is often far from unity, the effect of the threshold resummation is less pronounced.

\section{Conclusions}

In this paper we have presented a phenomenological application of the combined threshold and threshold-improved transverse momentum resummation introduced in ref. [16] for transverse momentum spectra. This combined resummation allows for an improvement of the transverse momentum distribution that holds for all values of momenta.

We have presented in section 2 various analytical frameworks needed for the phenomenology studies. A particular attention was focused on the implementation of the Borel method as an alternative prescription to perform the inverse Fourier and Mellin transform. It was shown that the Borel prescription owes good numerical stability while providing complete control of the power correction terms, introduced by the thresholdimproved $p_{T}$ resummation procedure, allowing us to reproduce standard $p_{T}$ results in the limit $p_{T} \rightarrow 0$. The results of our phenomenological studies were presented in section 3 

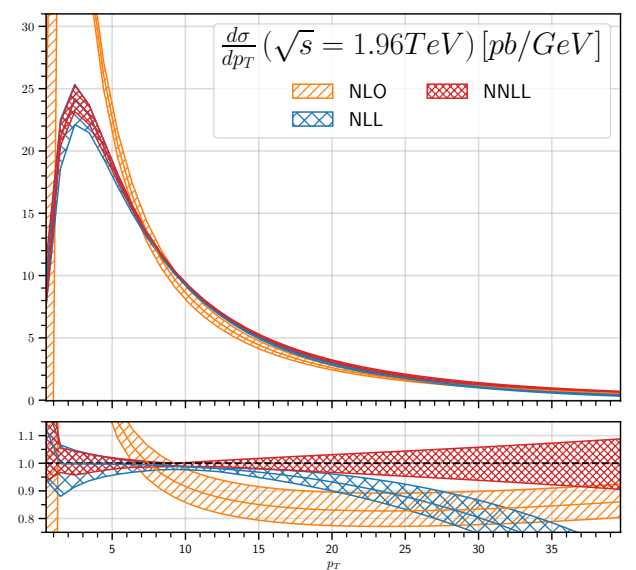

(a) SSpT resummation
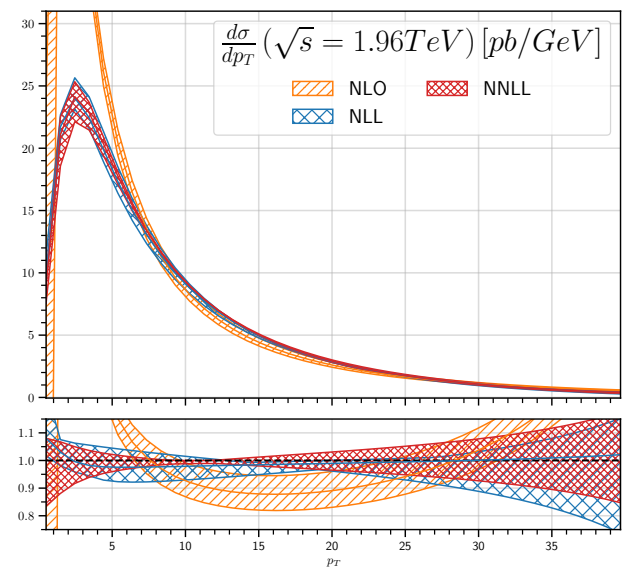

(c) TIpT resummation

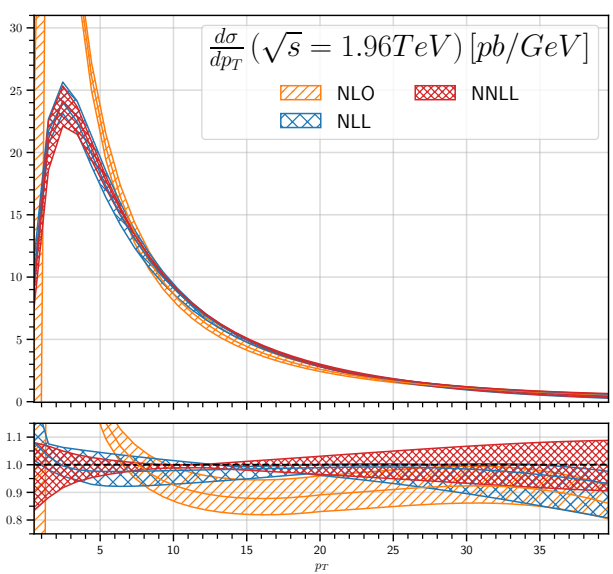

(e) combined resummation
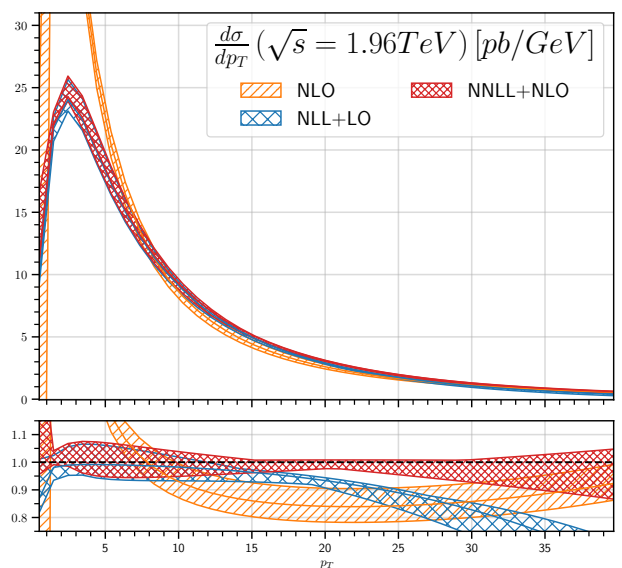

(b) SSpT resummation with matching

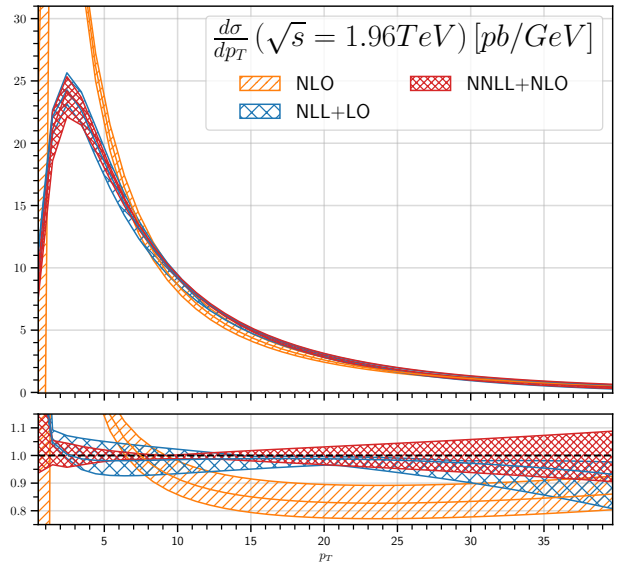

(d) TIpT resummation with matching
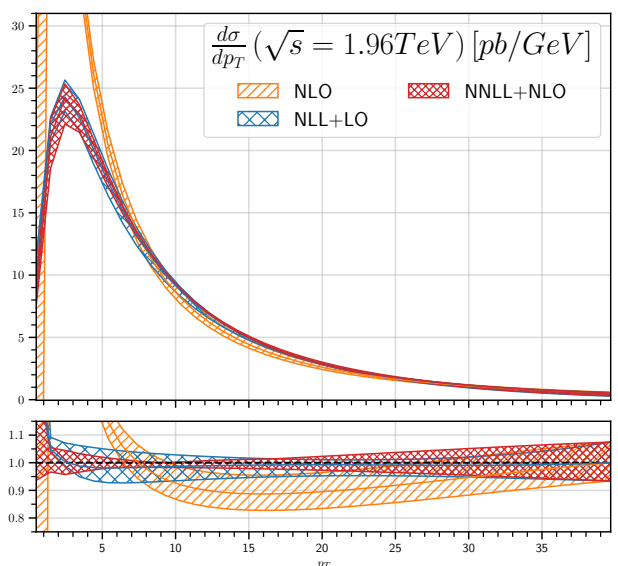

(f) combined resummation with matching

Figure 6. $p_{T}$ spectrum of the $Z$ boson production for various types of resummation: standard small- $p_{T}$ (top), threshold-improved small- $p_{T}$ (middle), and combined (bottom). The left plots show the pure resummation results while those matched to the fixed-order are shown on the right. The top panels compare the NLL and NNLL resummed results to the NLO. The lower panels show the ratio w.r.t. to the central value of the respective NNLL results. The uncertainty bands are computed by varying $\mu_{R}$ and $\mu_{F}$ using the 7-point method; in all cases, $Q=m_{Z}$. 
where we matched our resummed expression with fixed-order calculations. We found that the effects of the threshold-improved $p_{T}$ resummation have a modest significance for a $Z$ boson production via DY mechanism at small and moderate $p_{T}$ while sizeable improvement can be seen in the tail of the distribution. The difference, however, is rather significant in the case of Higgs boson produced via gluon fusion, which highlights the potential relevance of threshold resummation to gluon-induced processes [47-50]. As a main result we found that while threshold-improved $p_{T}$ resummation enhances the convergence at small- $p_{T}$, its combination with threshold resummation improves the agreement with fixed-order results in the intermediate and large- $p_{T}$ regions.

As possible directions for future development of this work, we will extend the result in ref. [16] to N3LL and interface our implementation with Monte Carlo codes in order to produce N3LL+NNLO predictions. In addition, it will be interesting to study further the relation between threshold and the modified $p_{T}$ resummation in order to identify the missing soft logarithms with an ultimate goal of developing a combined expression that does not rely on a profile matching function. This, in turn, can be extended to account for the high-energy (or small- $x$ ) resummation, which quite recently has been jointly performed with small- $p_{T}[51]$ and threshold resummation [32].

\section{Acknowledgments}

The author wish to thank Stefano Forte and Claudio Muselli for useful discussion on combined resummation, and Giancarlo Ferrera for providing help on standard $p_{T}$ resummation. We also express our deepest thanks to Juan M. Cruz-Martinez for the fixed-order results, Stefano Forte and Christopher Schwan for careful readings of the manuscript, and Luca Rottoli for several discussions. This work is supported by the European Research Council under the European Union's Horizon 2020 research and innovation Programme (grant agreement n. 740006).

Open Access. This article is distributed under the terms of the Creative Commons Attribution License (CC-BY 4.0), which permits any use, distribution and reproduction in any medium, provided the original author(s) and source are credited.

\section{References}

[1] W. Bizoń, P.F. Monni, E. Re, L. Rottoli and P. Torrielli, Momentum-space resummation for transverse observables and the Higgs $p_{\perp}$ at $N^{3} L L+N N L O$, JHEP 02 (2018) 108 [arXiv: 1705.09127] [INSPIRE].

[2] W. Bizoń et al., Fiducial distributions in Higgs and Drell-Yan production at $N^{3} L L+N N L O, J H E P 12$ (2018) 132 [arXiv:1805.05916] [INSPIRE].

[3] X. Chen et al., Precise QCD Description of the Higgs Boson Transverse Momentum Spectrum, Phys. Lett. B $\mathbf{7 8 8}$ (2019) 425 [arXiv: 1805.00736] [INSPIRE].

[4] W. Bizoń et al., The transverse momentum spectrum of weak gauge bosons at $N^{3} L L+N N L O$, Eur. Phys. J. C 79 (2019) 868 [arXiv:1905.05171] [INSPIRE]. 
[5] M.A. Ebert, J.K.L. Michel, I.W. Stewart and F.J. Tackmann, Drell-Yan $q_{T}$ Resummation of Fiducial Power Corrections at $N^{3} L L$, arXiv:2006.11382 [INSPIRE].

[6] T. Becher and M. Hager, Event-Based Transverse Momentum Resummation, Eur. Phys. J. C 79 (2019) 665 [arXiv:1904.08325] [INSPIRE].

[7] I. Scimemi and A. Vladimirov, Non-perturbative structure of semi-inclusive deep-inelastic and Drell-Yan scattering at small transverse momentum, JHEP 06 (2020) 137 [arXiv: 1912.06532] [INSPIRE].

[8] S. Kallweit, E. Re, L. Rottoli and M. Wiesemann, Accurate single- and double-differential resummation of colour-singlet processes with MATRIX+RadISH: $W^{+} W^{-}$production at the $L H C$, arXiv:2004.07720 [INSPIRE].

[9] M. Wiesemann, L. Rottoli and P. Torrielli, The $Z \gamma$ transverse-momentum spectrum at $N N L O+N^{3} L L$, Phys. Lett. B 809 (2020) 135718 [arXiv: 2006.09338] [inSPIRE].

[10] H.-n. Li, Unification of the $k_{T}$ and threshold resummations, Phys. Lett. B 454 (1999) 328 [hep-ph/9812363] [INSPIRE].

[11] E. Laenen, G.F. Sterman and W. Vogelsang, Recoil and threshold corrections in short distance cross-sections, Phys. Rev. D 63 (2001) 114018 [hep-ph/0010080] [InSPIRE].

[12] A. Kulesza, G.F. Sterman and W. Vogelsang, Joint resummation for Higgs production, Phys. Rev. D 69 (2004) 014012 [hep-ph/0309264] [INSPIRE].

[13] A. Kulesza, G.F. Sterman and W. Vogelsang, Joint resummation in electroweak boson production, Phys. Rev. D 66 (2002) 014011 [hep-ph/0202251] [INSPIRE].

[14] S. Marzani and V. Theeuwes, Vector boson production in joint resummation, JHEP 02 (2017) 127 [arXiv : 1612.01432] [INSPIRE].

[15] G. Lustermans, W.J. Waalewijn and L. Zeune, Joint transverse momentum and threshold resummation beyond NLL, Phys. Lett. B 762 (2016) 447 [arXiv:1605.02740] [INSPIRE].

[16] C. Muselli, S. Forte and G. Ridolfi, Combined threshold and transverse momentum resummation for inclusive observables, JHEP 03 (2017) 106 [arXiv:1701.01464] [INSPIRE].

[17] G. Parisi and R. Petronzio, Small Transverse Momentum Distributions in Hard Processes, Nucl. Phys. B 154 (1979) 427 [INSPIRE].

[18] G. Curci, M. Greco and Y. Srivastava, QCD Jets From Coherent States, Nucl. Phys. B 159 (1979) 451 [INSPIRE].

[19] Y.L. Dokshitzer, D. Diakonov and S.I. Troian, Hard Processes in Quantum Chromodynamics, Phys. Rept. 58 (1980) 269 [INSPIRE].

[20] J.C. Collins and D.E. Soper, Back-To-Back Jets: Fourier Transform from B to K-Transverse, Nucl. Phys. B 197 (1982) 446 [InSPIRE].

[21] G.F. Sterman, Summation of Large Corrections to Short Distance Hadronic Cross-Sections, Nucl. Phys. B 281 (1987) 310 [InSPIRE].

[22] J.C. Collins, D.E. Soper and G.F. Sterman, Transverse Momentum Distribution in Drell-Yan Pair and $W$ and $Z$ Boson Production, Nucl. Phys. B 250 (1985) 199 [INSPIRE].

[23] J.C. Collins, D.E. Soper and G.F. Sterman, Factorization of Hard Processes in QCD, Adv. Ser. Direct. High Energy Phys. 5 (1989) 1 [hep-ph/0409313] [INSPIRE]. 
[24] S. Catani and L. Trentadue, Resummation of the QCD Perturbative Series for Hard Processes, Nucl. Phys. B 327 (1989) 323 [InSPIRE].

[25] S. Catani, D. de Florian and M. Grazzini, Universality of nonleading logarithmic contributions in transverse momentum distributions, Nucl. Phys. B 596 (2001) 299 [hep-ph/0008184] [INSPIRE].

[26] S. Albino and R.D. Ball, Soft resummation of quark anomalous dimensions and coefficient functions in MS-bar factorization, Phys. Lett. B 513 (2001) 93 [hep-ph/0011133] [InSPIRE].

[27] E. Laenen, Resummation for observables at TeV colliders, Pramana 63 (2004) 1225 [INSPIRE].

[28] D. de Florian, A. Kulesza and W. Vogelsang, Threshold resummation for high-transverse-momentum Higgs production at the LHC, JHEP 02 (2006) 047 [hep-ph/0511205] [INSPIRE].

[29] G. Bozzi, S. Catani, D. de Florian and M. Grazzini, Transverse-momentum resummation and the spectrum of the Higgs boson at the LHC, Nucl. Phys. B 737 (2006) 73 [hep-ph/0508068] [INSPIRE].

[30] D. de Florian, G. Ferrera, M. Grazzini and D. Tommasini, Transverse-momentum resummation: Higgs boson production at the Tevatron and the LHC, JHEP 11 (2011) 064 [arXiv: 1109.2109] [INSPIRE].

[31] M. Bonvini, S. Forte and G. Ridolfi, Borel resummation of transverse momentum distributions, Nucl. Phys. B 808 (2009) 347 [arXiv:0807.3830] [INSPIRE].

[32] M. Bonvini, S. Forte and G. Ridolfi, Soft gluon resummation of Drell-Yan rapidity distributions: Theory and phenomenology, Nucl. Phys. B 847 (2011) 93 [arXiv:1009.5691] [INSPIRE].

[33] M. Bonvini, S. Forte, G. Ridolfi and L. Rottoli, Resummation prescriptions and ambiguities in SCET vs. direct QCD: Higgs production as a case study, JHEP 01 (2015) 046 [arXiv: 1409.0864] [INSPIRE].

[34] S. Forte and G. Ridolfi, Renormalization group approach to soft gluon resummation, Nucl. Phys. B 650 (2003) 229 [hep-ph/0209154] [INSPIRE].

[35] M. Bonvini, S. Forte, M. Ghezzi and G. Ridolfi, Threshold Resummation in SCET vs. Perturbative QCD: An Analytic Comparison, Nucl. Phys. B 861 (2012) 337 [arXiv: 1201.6364] [INSPIRE].

[36] G. Bozzi, S. Catani, G. Ferrera, D. de Florian and M. Grazzini, Production of Drell-Yan lepton pairs in hadron collisions: Transverse-momentum resummation at next-to-next-to-leading logarithmic accuracy, Phys. Lett. B 696 (2011) 207 [arXiv: 1007.2351] [INSPIRE].

[37] S. Catani, M.L. Mangano, P. Nason and L. Trentadue, The Resummation of soft gluons in hadronic collisions, Nucl. Phys. B 478 (1996) 273 [hep-ph/9604351] [INSPIRE].

[38] S. Forte, G. Ridolfi, J. Rojo and M. Ubiali, Borel resummation of soft gluon radiation and higher twists, Phys. Lett. B 635 (2006) 313 [hep-ph/0601048] [INSPIRE].

[39] R. Abbate, S. Forte and G. Ridolfi, A New prescription for soft gluon resummation, Phys. Lett. B 657 (2007) 55 [arXiv:0707.2452] [INSPIRE]. 
[40] C. Muselli, Resummation of transverse momentum distributions, Ph.D. Thesis, Università degli studi di Milano, Institutional Research Information System (IRIS) (2017).

[41] M. Bonvini, Resummation of soft and hard gluon radiation in perturbative QCD, Ph.D. Thesis, Genoa U. (2012) [arXiv: 1212.0480] [InSPIRE].

[42] A. Vogt, Efficient evolution of unpolarized and polarized parton distributions with QCD-PEGASUS, Comput. Phys. Commun. 170 (2005) 65 [hep-ph/0408244] [INSPIRE].

[43] NNPDF collaboration, Parton distributions for the LHC Run II, JHEP 04 (2015) 040 [arXiv: 1410.8849] [INSPIRE].

[44] A. Buckley et al., LHAPDF6: parton density access in the LHC precision era, Eur. Phys. J. C 75 (2015) 132 [arXiv:1412.7420] [INSPIRE].

[45] J.M. Cruz-Martinez, Next-to-Next-to-Leading Order QCD Corrections to Higgs Boson Production in Association with two Jets in Vector Boson Fusion, Ph.D. Thesis, Durham U. (2018) [INSPIRE].

[46] G. Bozzi, S. Catani, G. Ferrera, D. de Florian and M. Grazzini, Transverse-momentum resummation: A Perturbative study of $Z$ production at the Tevatron, Nucl. Phys. B $\mathbf{8 1 5}$ (2009) 174 [arXiv:0812.2862] [INSPIRE].

[47] D. Appell, G.F. Sterman and P.B. Mackenzie, Soft Gluons and the Normalization of the Drell-Yan Cross-section, Nucl. Phys. B 309 (1988) 259 [INSPIRE].

[48] W. Giele et al., The QCD/SM working group: Summary report, in 2nd Les Houches Workshop on Physics at TeV Colliders, pp. 275-426 (2002) [hep-ph/0204316] [INSPIRE].

[49] S. Catani, D. de Florian, M. Grazzini and P. Nason, Soft gluon resummation for Higgs boson production at hadron colliders, JHEP 07 (2003) 028 [hep-ph/0306211] [INSPIRE].

[50] V. Ravindran, J. Smith and W.L. van Neerven, NNLO corrections to the total cross-section for Higgs boson production in hadron hadron collisions, Nucl. Phys. B 665 (2003) 325 [hep-ph/0302135] [INSPIRE].

[51] S. Marzani, Combining $Q_{T}$ and small-x resummations, Phys. Rev. D 93 (2016) 054047 [arXiv:1511.06039] [INSPIRE]. 